\title{
String structures associated to indefinite Lie groups
}

\author{
Hisham Sati ${ }^{1}$ and Hyung-bo Shim ${ }^{2}$ \\ ${ }^{1}$ Division of Science and Mathematics, New York University Abu Dhabi, Saadiyat Island, Abu Dhabi, UAE \\ ${ }^{2}$ Department of Mathematics, University of Pittsburgh, Pittsburgh, PA 15260, USA
}

April 2, 2019

\begin{abstract}
String structures have played an important role in algebraic topology, via elliptic genera and elliptic cohomology, in differential geometry, via the study of higher geometric structures, and in physics, via partition functions. We extend the description of String structures from connected covers of the definite-signature orthogonal group $\mathrm{O}(n)$ to the indefinite-signature orthogonal group $\mathrm{O}(p, q)$, i.e. from the Riemannian to the pseudo-Riemannian setting. This requires that we work at the unstable level, which makes the discussion more subtle than the stable case. Similar, but much simpler, constructions hold for other noncompact Lie groups such as the unitary group $\mathrm{U}(p, q)$ and the symplectic group $\operatorname{Sp}(p, q)$. This extension provides a starting point for an abundance of constructions in (higher) geometry and applications in physics.
\end{abstract}

\section{Contents}

1 Introduction $\quad 2$

2 The Postnikov tower, Whitehead tower, and variants 4

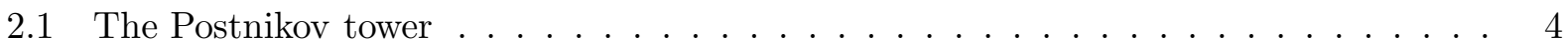

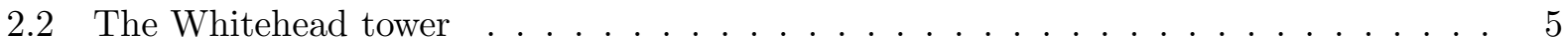

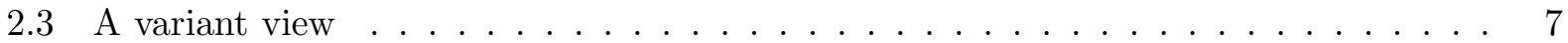

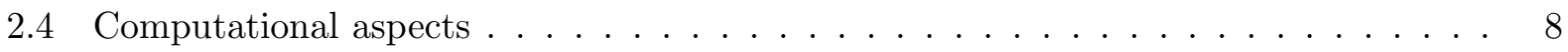

2.5 Special case: higher connected covers of classifying spaces and obstructions . . . . 10

3 Applications 13

3.1 Special orthogonal groups $\mathrm{SO}(n)$ and $\mathrm{SO}(p, q) \ldots \ldots \ldots \ldots \ldots$

3.2 Indefinite Spin groups . . . . . . . . . . . . . . . . . . . . 14

3.3 Indefinite String groups . . . . . . . . . . . . . . . . . . 17

3.4 String structure associated to indefinite unitary and symplectic groups . . . . . . . 21

3.5 Relation to twisted structures . . . . . . . . . . . . . . . . 23 


\section{Introduction}

Lie groups play an important role in characterizing symmetries. Picking the appropriate Lie group allows for certain structures to be unambiguously defined. For example, a Riemannian structure on a manifold requires the principal frame bundle corresponding to the tangent bundle to have an orthogonal group as a structure group. To talk about orientations one needs the special orthogonal group, and to properly discuss spinors one needs to lift to the double cover, which is the Spin group. All of these structures are low degree phenomena which can be encoded uniformly and succinctly via the Whitehead tower of the orthogonal group (see [30] 31] [29]).

In algebraic topology, one usually studies the Whitehead tower of the stable orthogonal group (see [30]). In particular, killing the third homotopy group leads to the stable String group (see [34 [35]). There are many constructions and applications associated with String structures and to the String group in various areas of mathematics and physics. The following is a sample which is necessarily incomplete. In algebraic topology, String structures play a role of orientation for elliptic cohomology [1] 34] 35]. In differential geometry, String connections play a role in geometrically describing bundles with the String group as a structure group [31] [26] [39] [6] [7]. In mathematical physics, conditions for having String structures arise as anomaly cancellation conditions [16] [30] 31].

This paper aims to initiate a new angle on the subject. We will be interested in the case of the indefinite orthogonal group $\mathrm{O}(p, q)$, which from a geometric point of view can be viewed as the structure group of the tangent bundle of a pseudo-Riemannian manifold of dimension $n=p+q$. As in the Riemannian case, one is interested in considering oriented and then Spin pseudo-Riemannain manifolds and for that one needs to lift $\mathrm{O}(p, q)$ to appropriate groups $\mathrm{SO}(p, q)$ and $\operatorname{Spin}(p, q)$, respectively. This involves a lot of subtleties and unlike the Riemannian case, here it is a priori not obvious which homotopy (sub-)groups to kill in order to get to the appropriate covering group. We spend some time discussing this before embarking on considering the corresponding indefinite String groups. In geometry and physics, the cases $p=1,2$ are particularly interesting, as these correspond to the Lorentz group and the conformal group, respectively. We address all cases, and for the most part there is a natural split into cases $p=1, p=2$ and $p \geq 3$.

Another subtle matter in our discussion is that we need to work in the unstable range for the orthogonal groups, that is with $\mathrm{O}(n)$ for finite $n$, i.e. without taking a limit on the rank as usually is done in the literature. For the Riemannian case, the Whitehead tower (cf. [30]) would be replaced by one involving finite $n$

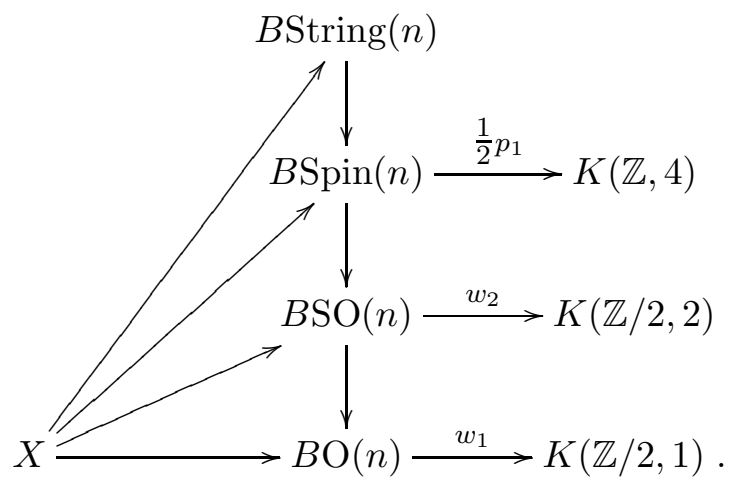


where $w_{1} \in H^{1}(B \mathrm{O}(n) ; \mathbb{Z} / 2)$ and $w_{2} \in H^{2}(B \mathrm{SO}(n) ; \mathbb{Z} / 2)$ are the universal first and second StiefelWhitney classes, while $\frac{1}{2} p_{1} \in H^{4}(B \operatorname{Spin}(n) ; \mathbb{Z})$ is the universal first Spin characteristic class.

This gives a homotopy theoretical construction of an 8-connected cover $(B \mathrm{O}(n))\langle 8\rangle$ of the classifying space of $\mathrm{O}(n)$. Having a classifying space points to presence of a group. Indeed, Stolz [34] constructs a topological group $\widehat{G}$ as an extension

$$
1 \longrightarrow \operatorname{Gauge}(P) \longrightarrow \widehat{G} \longrightarrow \operatorname{Spin}(n) \longrightarrow 1
$$

where $P \rightarrow \operatorname{Spin}(n)$ is the $P \mathrm{U}(\mathcal{H})$-principal bundle with $\mathcal{H}$ an infinite-dimensional separable Hilbert space such that $B \widehat{G}$ is the 8-connected cover of $B \mathrm{O}(n)$. This topological group $\widehat{G}$ is homotopy equivalent to the String group String $(n)$, defined via the fibration $K(\mathbb{Z}, 2) \rightarrow \operatorname{String}(n) \rightarrow \operatorname{Spin}(n)$, so that $\operatorname{String}(n)$ has a group structure. This also has a differentiable structure [23]. Since then many models of the String group have appeared, each having different desirable features (see e.g. the appendix of [7] for seven such models). For instance, in one model [23], $\operatorname{String}(n)$ is constructed as an extension as in eq. (1.2) of Lie groups so that $\widehat{G}$ has a Fréchet-Lie group structure uniquely determined up to isomorphism. The presence of such structures allows us to talk about $\operatorname{String}(n)$ principal bundles and String manifolds, regarding $\operatorname{String}(n)$ as a structure group.

Studying the Spin group and its classifying space from the cohomology point of view requires understanding of the first generator, i.e., the generator in degree three of the cohomology of the Spin group or the generator in degree four of the corresponding classifying space (see [40] [7] for interesting relations between the two). It is known from [36] that the cohomology ring of $B$ Spin in the stable case is generated by the Spin characteristic classes, the degree four generator of which is $\frac{1}{2} p_{1}$. However, we are interested in the unstable case, and indeed it was shown by McLaughlin [21] that $\frac{1}{2} p_{1}$ is also the generator of the cohomology $H^{4}(B \operatorname{Spin}(n) ; \mathbb{Z})$. The lifting of the structure group from $\operatorname{Spin}(n)$ to $\operatorname{String}(n)$ of a bundle over a manifold $X$ is then possible when the obstruction $\frac{1}{2} p_{1}(X)$ vanishes. We will be interested in generalizing this result to the case of $B \operatorname{Spin}(p, q)$.

String structures are interesting from the geometric point of view due to the relation between the Riemannian geometry of a manifold and characteristic classes associated with String structures on that manifold. The Stolz-Höhn conjecture says the following: Let $X$ be a smooth closed string manifold of dimension $4 k$. If $X$ admits a Riemannian metric with positive Ricci curvature, then the Witten genus $\phi_{W}(X)$ vanishes.

Homotopy-theoretically, String structures amount to having a String orientation, which in relation to modular forms gives a corresponding orientation to the spectrum of topological modular forms (tmf), MString $\rightarrow$ tmf [1]. Conjecturally, the Witten genus is constructed as an index of a Dirac operator on loop space [44]. This is the String analog to the theorem on vanishing $\hat{A}$-genus by Lichnerowicz [19] for Spin manifolds: Let $X$ be a smooth closed spin manifold of dimension $2 k$. If $X$ admits a Riemannian affine connection with nonnegative and not identically zero Riemannian curvature, then the $\hat{A}$-genus vanishes.

The Atiyah-Bott-Shapiro map $M$ Spin $\rightarrow K \mathrm{O}$ is constructed using the representations of the Spin groups, and relies on knowing that for a space $X$, elements of $K \mathrm{O}^{0}(X)$ are represented by vector bundles over $X$. It gives a $K$ O-theory Thom isomorphism for Spin-vector bundles, and is a topological expression for the index of the Dirac operator. We hope that similar questions can be explored in the semi-Riemannian setting. 
This paper is organized as follows. We start with a more general setting of the problem that we hope explain some of the homotopy theoretical constructions encountered here as well as in previous literature. In Sec. 2.1 and 2.2, we describe the Postnikov tower and the Whitehead tower of a space in a manner that is appropriate for applications. Then in Sec. 2.3 we provide a variant point of view on the Whitehead tower in way of clarification. Since lifts of indefinite Lie groups will be determined by their maximal compact subgroups, which are products, we discuss in Sec. 2.4 useful conditions on behavior of cohomology of products. The discussion is needed since we work with integral cohomology. This is applied in Sec. 2.5 to classifying spaces, where we identify the obstructions and where the fibrations become fiber bundles. We start with the applications in Sec. 3. First, in order to make sure we are on firm ground, we discuss the indefinite orthogonal groups in Sec. 3.1, highlighting their unstable homotopy groups. A subtle issue in the non-vanishing of the fundamental group of the corresponding Spin groups is addressed in Sec. 3.2, This is used in Sec. 3.3 to define the indefinite String groups, where we identify the obstructions explicitly by studying the generators of the classifying space of $B \operatorname{Spin}(n)$ in the unstable case. We then show in Sec. 3.4 how the definitions and constructions extend to the case of $\mathrm{U}(p, q)$ and $\operatorname{Sp}(p, q)$, where subtle issues with stability are absent. Finally, in Sec. 3.5 we describe relations to twisted structures, and end with highlighting future work that we hope to do.

\section{The Postnikov tower, Whitehead tower, and variants}

In this section we will provide a careful treatment of the towers arising in co-killing homotopy groups of Lie groups. The idea is that Postnikov towers arise when killing homotopy groups above a certain degree, while Whitehead towers arise when killing homotopy groups below a certain degree- hence the term co-killing. We will also provide a variant tower of higher connected covers, which we demonstrate is equivalent to the latter. We believe that such a treatment, while certainly known to experts, seems to be missing from existing literature to the best of our knowledge. This technical treatment, we hope, will be for the benefit of the reader and will make the paper self-contained. Readers not interested in these details might wish to skip this section.

\subsection{The Postnikov tower}

We start by recalling the Postnikov tower (see [13] [12]). We will take this as our starting point to connect to the Whitehead tower.

Theorem 2.1 (see [13] [12]) Let $X$ be a simple path-connected space with a map $\alpha: X \rightarrow X_{1}$, where $X_{1}$ is the Eilenberg-MacLane space $K\left(\pi_{1}(X), 1\right)$, that induces an isomorphism on fundamental groups $\pi_{1}(X) \rightarrow \pi_{1}\left(X_{1}\right)$. Then there are spaces $X_{n}$ with maps $\alpha_{n}: X \rightarrow X_{n}$ which induce isomorphisms on homotopy groups $\pi_{k}(X) \rightarrow \pi_{k}\left(X_{n}\right)$ for $k \leq n$ and $\pi_{k}\left(X_{n}\right)=0$ for $k>n$ with fibrations $p_{n+1}: X_{n+1} \rightarrow X_{n}$ such that $\alpha_{n}=p_{n+1} \circ \alpha_{n+1}$.

Assuming that such $\alpha_{n}: X \rightarrow X_{n}$ is given, the space $X_{n+1}$ will be defined as the homotopy fiber of a certain map $k^{n+2}: X_{n} \rightarrow K\left(\pi_{n+1}(X), n+2\right)$ that is equivalent to the corresponding cohomology class in $H^{n+2}\left(X_{n}, \pi_{n+1}(X)\right)$ such that the induced class $\alpha_{n}^{*} k^{n+2} \in H^{n+2}\left(X, \pi_{n+1}(X)\right)$ is trivial. By the universality of the homotopy fiber $X_{n+1}$, one then obtain the desired map $\alpha_{n+1}: X \rightarrow X_{n+1}$. 
These maps are assembled in the following diagram called the Postnikov tower of $X$ :

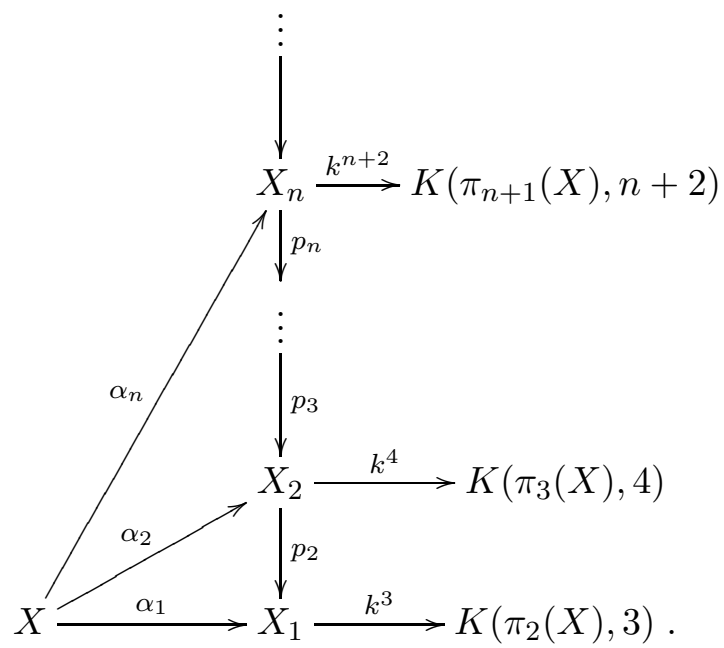

One could see how to choose such a map $k^{n+2}: X_{n} \rightarrow K\left(\pi_{n+1}(X), n+2\right)$ and how to obtain $X_{n+1}$ as the homotopy fiber explicitly as follows. Let $j: C\left(\alpha_{n}\right) \rightarrow K\left(\pi_{n+1}(X), n+2\right)$ be the inductive attachment of cells to the cofiber $C\left(\alpha_{n}\right)$ to match the homotopy group of the space $K\left(\pi_{n+1}(X), n+2\right)$ from the identification $\pi_{n+2}\left(C\left(\alpha_{n}\right)\right) \cong \pi_{n+1}(X)$ and the fact that $C\left(\alpha_{n}\right)$ is $(n+1)$-connected. On the other hand, we have the inclusion $X_{n} \hookrightarrow C\left(\alpha_{n}\right)$ and we take the composite $k^{n+2}: X_{n} \rightarrow K\left(\pi_{n+1}(X), n+2\right)$. From the inclusion of the cone $C(X)$ in the cofiber $C\left(\alpha_{n}\right), \chi_{x}(t):=j(x, 1-t)$ for $(x, 1-t) \in C(X)$ is in the path space $P K\left(\pi_{n+1}(X), n+2\right)$. This then makes the outer rectangle part of the following pullback diagram

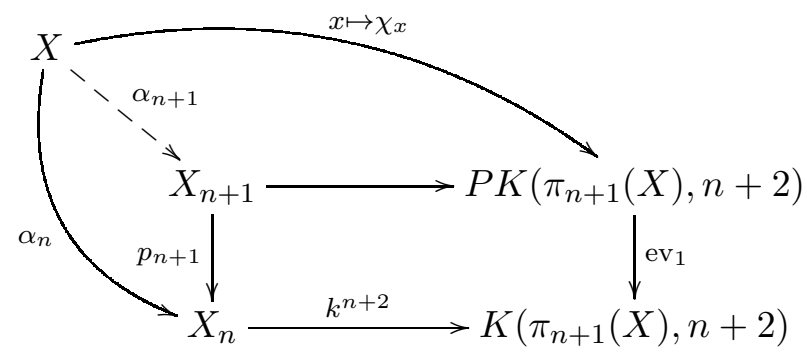

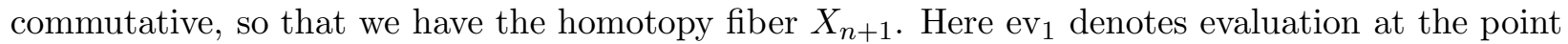
1 of the interval in the path space. Note that $P K\left(\pi_{n+1}(X), n+2\right)$ is a homotopy equivalent to a point space so the commutativity of the diagram implies that the cohomology class $\alpha_{n}^{*} k^{n+2} \in$ $H^{n+2}\left(X ; \pi_{n+1}(X)\right)$ is trivial. Therefore, we obtain the map $\alpha_{n+1}$ which satisfies $\alpha_{n}=p_{n+1} \circ \alpha_{n+1}$ and induces an isomorphism $\pi_{k}(X) \rightarrow \pi_{k}\left(X_{n+1}\right)$ for $k \leq n+1$ and makes $X_{n+1}$ have trivial $k^{\text {th }}$ homotopy group for $k>n+2$. By inductive application of this process, one obtains the Postnikov tower of $X$.

\subsection{The Whitehead tower}

Next we consider the Whitehead tower, which is in a sense a dual to the Postnikov tower [42] 43. Note that the Postnikov tower approximates the homotopy groups of $X$ "from the bottom" in the 
sense that it admits an isomorphism of lower homotopy groups with higher homotopy groups being killed. One then may seek the dual process of estimating the homotopy groups of $X$ "from the top", in the sense that lower homotopy groups are killed while higher homotopy groups are isomorphic to those of $X$.

Theorem 2.2 (See [43]) Let $X$ be a (path) connected space with a Postnikov tower. Then there are (path) connected spaces $X\langle n\rangle$ such that $\pi_{k}(X\langle n\rangle)=0$ for $k \leq n$ and maps $\widehat{\alpha}_{n}: X\langle n\rangle \rightarrow X$ that induce isomorphisms $\pi_{k}(X\langle n\rangle) \rightarrow \pi_{k}(X)$ for $k>n$. Moreover, there is a fibration $\widehat{p}_{n+1}$ : $X\langle n+1\rangle \rightarrow X\langle n\rangle$ for each $n$ such that $\widehat{\alpha}_{n+1}=\widehat{\alpha}_{n} \circ \widehat{p}_{n+1}$ with a fiber the based loop space $\Omega X\langle n\rangle$.

From the Postnikov tower of $X$, we have spaces $X_{n}$ and maps $\alpha_{n}: X \rightarrow X_{n}$. By taking the homotopy fiber $X\langle n\rangle$ of $\alpha_{n}$, we obtain a fibration $\widehat{\alpha}_{n}: X\langle n\rangle \rightarrow X$ that induces isomorphisms $\pi_{k}(X\langle n\rangle) \rightarrow \pi_{k}(X)$ for $k>n$ and makes $\pi_{k}(X\langle n\rangle)=0$ for $k \leq n$. Since $X\langle n+1\rangle \rightarrow X$ factors through $X\langle n\rangle$ naturally as in the diagram

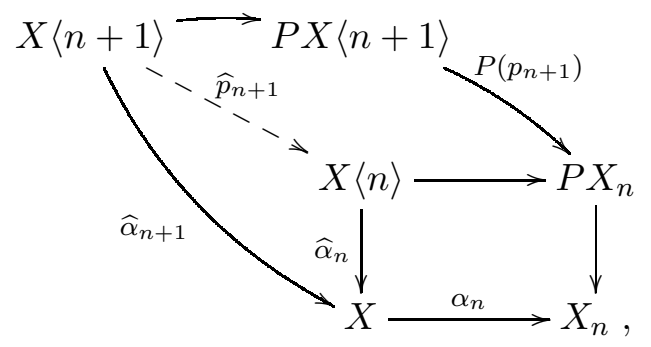

we obtain a map $\widehat{p}_{n+1}: X\langle n+1\rangle \rightarrow X\langle n\rangle$, which can be made into a fibration, up to homotopy equivalence, such that $\widehat{\alpha}_{n+1}=\widehat{\alpha}_{n} \circ \widehat{p}_{n+1}$. The induced long exact sequence of the fibration $\widehat{p}_{n+1}$ shows that the fiber is homotopy equivalent to the Eilenberg-MacLane space $K\left(\pi_{n+1}(X), n\right)$. The tower of fibrations thus obtained is a dual version of the Postnikov tower called the Whitehead tower [42] [43]:

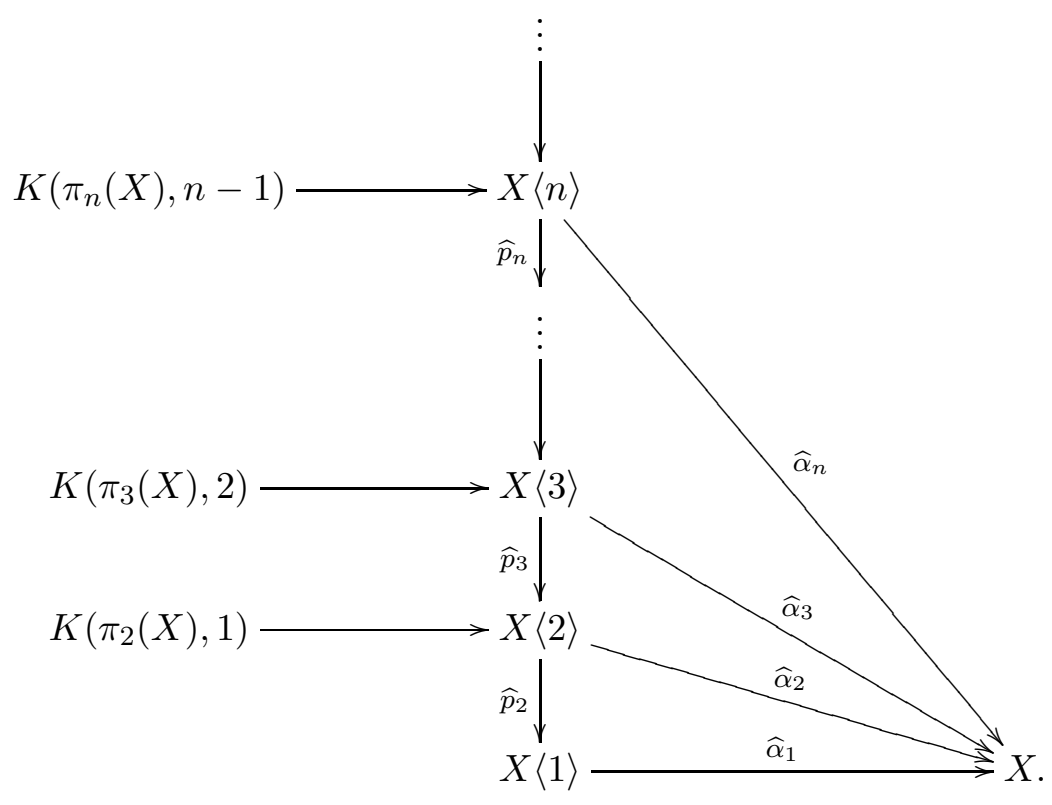




\subsection{A variant view}

Each fibration $\widehat{p}_{n+1}: X\langle n+1\rangle \rightarrow X\langle n\rangle$ in the Whitehead tower can be regarded as the $(n+1)$ connected covering of $X\langle n\rangle$ and we may ask the "lifting" question, i.e. under what circumstance can a map $M \rightarrow X\langle n\rangle$ be lifted to $M \rightarrow X\langle n+1\rangle$ as in the commutative diagram

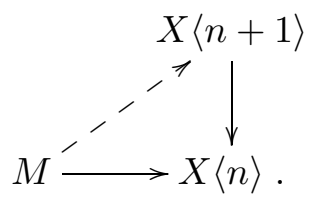

To answer this question we need the following important property of the sequence of spaces appearing in the Whitehead tower.

Proposition 2.3 Let $X$ be a connected space. Then there are spaces $X\langle n\rangle$, where $X\langle 1\rangle:=X$, with fibrations $\hat{p}_{n+1}: X\langle n+1\rangle \rightarrow X\langle n\rangle$ with fiber $K\left(\pi_{n}(X), n-1\right)$ such that

$$
\pi_{k}(X\langle n\rangle) \cong \begin{cases}0 & \text { for } k<n \\ \pi_{k}(X) & \text { for } k \geq n\end{cases}
$$

Remark 1 These spaces $X\langle n+1\rangle$ are constructed as the homotopy fibers of a cohomology class $\lambda_{n} \in H^{n}\left(X\langle n\rangle ; \pi_{n}(X)\right)$ that induces an isomorphism $\pi_{n}(X\langle n\rangle) \stackrel{\cong}{\rightarrow} \pi_{n}(X)$, and a map $f: M \rightarrow$ $X\langle n\rangle$ is lifted to $\widetilde{f}: M \rightarrow X\langle n+1\rangle$ if the induced cohomology class $f^{*} \lambda_{n} \in H^{n}\left(M ; \pi_{n}(X)\right)$ is trivial.

Thus we have the following diagram that one might call the tower of higher connected covers

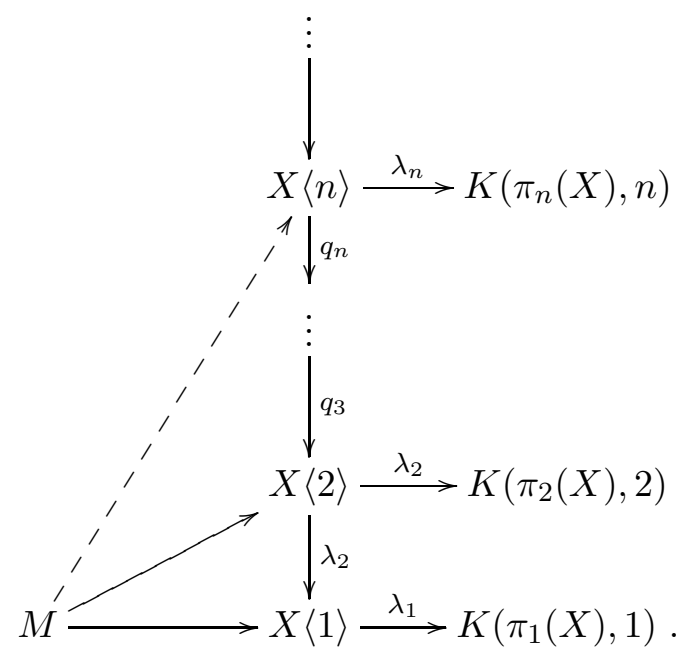

The construction is an inductive application of the process in the following lemma.

Lemma 2.4 (Killing $n^{\text {th }}$ homotopy group) Let $X$ be a simply connected space and $Y$ be an $(n-1)$-connected space whose cohomology class $\lambda_{n} \in H^{n}\left(Y ; \pi_{n}(X)\right)$ induces an isomorphism $\pi_{n}(Y) \rightarrow \pi_{n}(X)$. Then the homotopy fiber $\widehat{Y}$ of $\lambda_{n}$ satisfies

$$
\pi_{k}(\widehat{Y}) \cong \begin{cases}0 & \text { for } k=n \\ \pi_{k}(Y) & \text { for } k \neq n\end{cases}
$$


with a fibration $q: \widehat{Y} \rightarrow Y$. A map $f: M \rightarrow Y$ is lifted to $\widehat{f}: M \rightarrow \widehat{Y}$ with respect to the fibration $q$ when the induced cohomology class $f^{*} \lambda_{n} \in H^{n}\left(M ; \pi_{n}(X)\right)$ is trivial.

Proof. We would like to consider the lifting

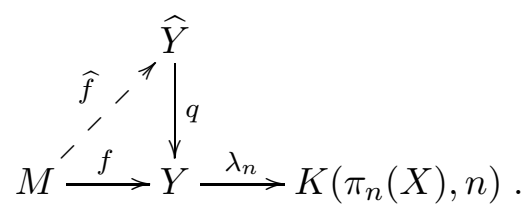

The result then follows from considering the following pullback diagram

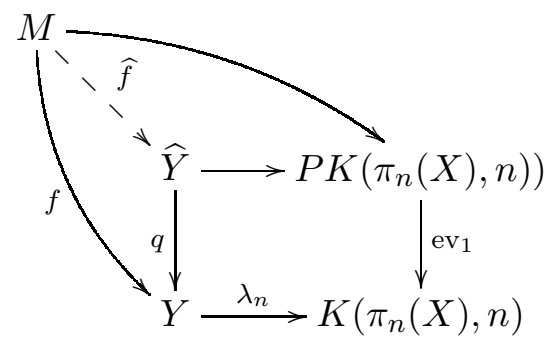

and its long exact sequence of homotopy groups and the fact that the path space $P K\left(\pi_{n}(X), n\right)$ is contractible. Here, the map $\mathrm{ev}_{1}$ is the evaluation map at the point 1 in the path.

Remark 2 (i) One way of figuring out that a given fibration $K\left(\pi_{n}(X), n-1\right) \rightarrow X\langle n+1\rangle \rightarrow X\langle n\rangle$ fits in the tower of higher connected covers is to check that a map $f: M \rightarrow X\langle n\rangle$ can be lifted up to a map $\widehat{f}: M \rightarrow X\langle n+1\rangle$ if and only if the cohomology class $f^{*} \lambda_{n} \in H^{n}\left(M ; \pi_{n}(X)\right)$ induced by $\lambda_{n}$ which induced the isomorphism of $n^{\text {th }}$-homotopy group vanishes.

(ii) It is convenient to rework the definition in a way making explicit the obstruction problem giving the lift from $X\langle n-1\rangle$ to $X\langle n\rangle$. Indeed, the two constructions, namely the Whitehead tower and the tower of higher connected covers, are equivalent by the homotopy commutative diagram:

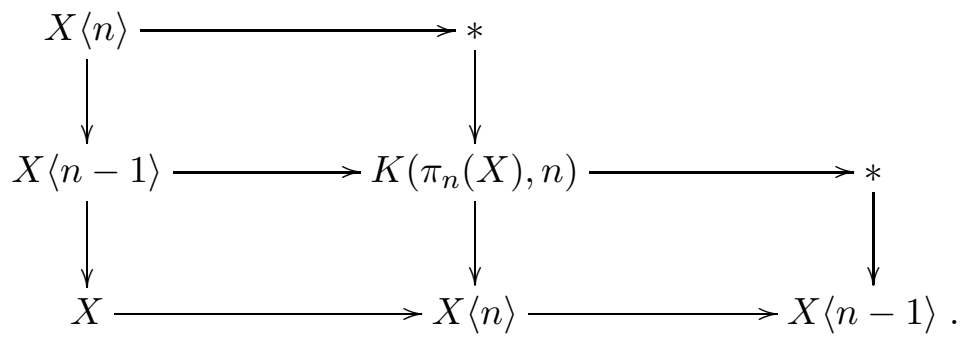

\subsection{Computational aspects}

We now turn to useful computational aspects of the above constructions. This will also be useful when considering indefinite Lie groups in Sec. 3. We would like to determine which classes in $H^{n}\left(Y ; \pi_{n}(X)\right)$, realized as homotopy classes of maps $Y \rightarrow K\left(\pi_{n}(X), n\right)$, induce isomorphisms $\pi_{n}(Y) \cong \pi_{n}(X)$. We do so by relating the summand $\operatorname{Hom}\left(H_{n}(Y, \mathbb{Z}), \pi_{n}(X)\right)$ in $H^{n}\left(Y ; \pi_{n}(X)\right)$ via the Hurewicz map $\pi_{n}(Y) \rightarrow H_{n}(Y, \mathbb{Z})$. In general, one needs strong assumptions on $Y$ in order for 
this to be viable, as the Hurewicz map could be zero. However, under the assumption that $Y$ is $(n-1)$-connected, we have an isomorphism $H^{n}\left(Y, \pi_{n}(X)\right) \cong \operatorname{Hom}\left(\pi_{n}(Y), \pi_{n}(X)\right)$.

Suppose that $n$th homotopy group of $Y$ is of the form $A \times B$ for some abelian groups $A$ and $B$ such that each of $H^{n}(Y ; A)$ and $H^{n}(Y ; B)$ is freely generated by a single generator $\alpha$ and $\beta$, respectively. We will denote by $(\alpha, 0)$ and $(0, \beta)$ the classes in $H^{n}(Y, A \times B)$ obtained from the corresponding classes in $H^{n}(Y ; A)$ and $H^{n}(Y ; B)$ via the canonical homomorphism $A \rightarrow A \times B$ and $B \rightarrow A \times B$, respectively. Then $\alpha$ and $\beta$ induce group homomorphism $\pi_{n}(X) \rightarrow A$ and $\pi_{n}(X) \rightarrow B$ so that neither $(\alpha, 0)$ nor $(0, \beta)$ in $H^{n}(Y ; A \times B)$ can induce an isomorphism $\pi_{n}(Y) \stackrel{\cong}{\rightrightarrows} A \times B$. However, the class in $H^{n}(Y ; A) \times H^{n}(Y ; B)$ inducing the isomorphism does exist and is of the form $(\tilde{\alpha}, \tilde{\beta})$ where $\tilde{\alpha}=a \alpha \in H^{n}(Y ; A)$ and $\tilde{\beta}=b \beta \in H^{n}(Y ; B)$ for some nonzero integers $a$ and $b$. At any rate, taking $Y$ is $(n-1)$-connected, we have an isomorphism

$$
\operatorname{Hom}_{\mathbb{Z}}\left(\pi_{n}(Y), A\right) \times \operatorname{Hom}_{\mathbb{Z}}\left(\pi_{n}(Y), A\right) \longrightarrow \operatorname{Hom}_{\mathbb{Z}}\left(\pi_{n}(Y), A \times B\right) .
$$

On the other hand, still assuming $\pi_{n}(X) \cong \pi_{n}(Y)$, if $Y$ is homotopy equivalent to the product space $Y^{\prime} \times Y^{\prime \prime}$ and

$$
H^{n}\left(Y ; \pi_{n}(X)\right) \cong H^{n}\left(Y^{\prime} ; \pi_{n}(X)\right) \times H^{n}\left(Y^{\prime \prime} ; \pi_{n}(X)\right)
$$

then we have the decomposition

$$
H^{n}\left(Y ; \pi_{n}(X)\right) \cong H^{n}\left(Y^{\prime} ; \pi_{n}\left(Y^{\prime}\right)\right) \times H^{n}\left(Y^{\prime} ; \pi_{n}\left(Y^{\prime \prime}\right)\right) \times H^{n}\left(Y^{\prime \prime} ; \pi_{n}\left(Y^{\prime}\right)\right) \times H^{n}\left(Y^{\prime \prime} ; \pi_{n}\left(Y^{\prime \prime}\right)\right) .
$$

This is immediately the case for our spaces, since they are assumed to be $(n-1)$-connected.

Assuming further that both abelian groups $H^{n}\left(Y^{\prime} ; \pi_{n}\left(Y^{\prime}\right)\right)$ and $H^{n}\left(Y^{\prime \prime} ; \pi_{n}\left(Y^{\prime \prime}\right)\right)$ are each cyclic with a single class generator $\alpha^{\prime}$ and $\alpha^{\prime \prime}$, respectively, the cohomology class $\left(\alpha^{\prime}, \alpha^{\prime \prime}\right)$ induces the desired isomorphism $\pi_{n}\left(Y^{\prime} \times Y^{\prime \prime}\right) \stackrel{\cong}{\rightrightarrows} \pi_{n}\left(Y^{\prime}\right) \times \pi_{n}\left(Y^{\prime \prime}\right)$. Obviously, the cohomology class $\left(\alpha^{\prime}, \alpha^{\prime \prime}\right)$ is represented by $\alpha^{\prime} \times \alpha^{\prime \prime}: Y^{\prime} \times Y^{\prime \prime} \rightarrow K\left(\pi_{n}\left(Y^{\prime}\right), n\right) \times K\left(\pi_{n}\left(Y^{\prime \prime}\right), n\right)$. Thus, we have obtained:

Proposition 2.5 For a path-connected and $(n-1)$-connected space $Y$ the homotopy fiber $\widehat{Y}$ of $\lambda_{n}: Y \rightarrow K\left(\pi_{n}(Y), n\right)$ has homotopy groups isomorphic to those of $Y$ except $\pi_{n}(\widehat{Y})=0$, according to the following cases:

(i) $\lambda_{n}=\alpha$ if $\alpha$ is the only generator of $H^{n}\left(Y, \pi_{n}(X)\right)$, i.e., we have a homotopy pullback diagram

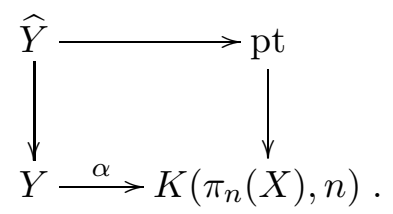

(ii) $\lambda_{n}=(\widehat{\alpha}, \widehat{\beta})$ if $\pi_{n}(Y) \cong A \times B$ and $H^{n}(Y ; A)$ and $H^{n}(Y ; B)$ are free with generators $\alpha$ and $\beta$, respectively, where $\widehat{\alpha}=a \alpha$ and $\widehat{\beta}=b \beta$ for some nonzero integers a and $b$, respectively, i.e.

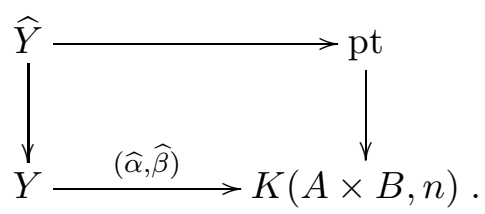


(iii) $\lambda_{n}=\alpha^{\prime} \times \alpha^{\prime \prime}$ which is equivalent to the map $Y^{\prime} \times Y^{\prime \prime} \rightarrow K\left(\pi_{n}\left(Y^{\prime}\right), n\right) \times K\left(\pi_{n}\left(Y^{\prime \prime}\right), n\right)$ if $Y \simeq Y^{\prime} \times Y^{\prime \prime}$ where $\alpha^{\prime}, \alpha^{\prime \prime}$ are the only generators of $H^{n}\left(Y^{\prime}, \pi_{n}\left(Y^{\prime}\right)\right)$ and $H^{n}\left(Y^{\prime \prime}, \pi_{n}\left(Y^{\prime \prime}\right)\right)$ respectively, and we again have the homotopy pullback diagram

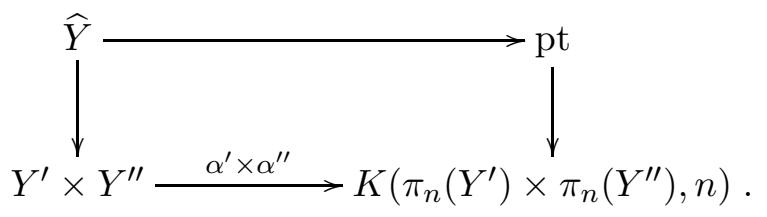

\subsection{Special case: higher connected covers of classifying spaces and obstructions}

We will mainly be interested in our spaces being topological groups. Let $G$ be an $(n-1)$-connected topological group. 1 Then $B G$ is a classifying space of $G$ such that $\pi_{i}(B G)=0$ for $i \leq n$. By taking the homotopy fiber of the canonical map $B G \rightarrow K\left(\pi_{n+1}(B G), n+1\right)$, we obtain a space $\widehat{B G}$ for which $\pi_{n+1}(\widehat{B G})$ is trivial. Then its loop space $\Omega \widehat{B G}$ satisfies $\pi_{n}(\Omega \widehat{B G})=0$. Setting $\widehat{G}:=\Omega \widehat{B G}$, we get a topological space which is $G$ with $\pi_{n}$ killed. The immediate consequence of this observation is the following:

Lemma 2.6 $\widehat{B G}$ is weakly homotopy equivalent to $B(\widehat{G})$.

Note that the statement of the lemma can be promoted to a homotopy equivalence provided $B(\widehat{G})$ is taken to be the usual classifying space of the (group-like) $A_{\infty}$-space $\widehat{G}$.

We now consider classification of the corresponding bundles. From the general discussion in previous sections we have the following results.

Proposition 2.7 Suppose that there is a G-principal bundle over $M$ determined by a classifying map $f: M \rightarrow B G$. Then there exists a map $\hat{f}: M \rightarrow B \widehat{G}$ providing a $\widehat{G}$-principal bundle over the same base $M$ compatible with the bundle $f$ with respect to the map $B \widehat{G} \rightarrow B G$ if $f^{*} \lambda_{n} \in H^{n+1}\left(M, \pi_{n}(G)\right)$ is trivial for some $\lambda_{n} \in H^{n+1}\left(B G ; \pi_{n}(G)\right)$ that induces an isomorphism $\pi_{n}(B G) \rightarrow \pi_{n-1}(G)$.

Remark 3 (i) The condition in Proposition (2.7) is equivalent to saying that $f^{*} \lambda_{n}$ factors through a point space up to homotopy:

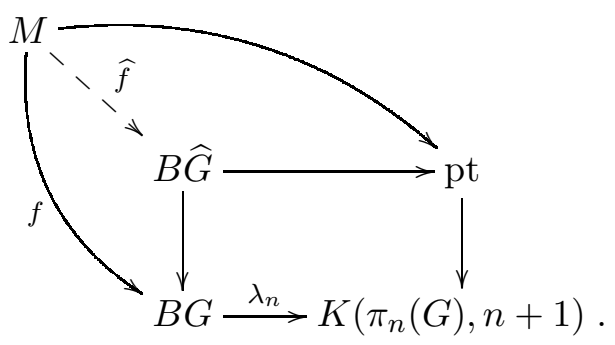

(ii) We say that the obstruction $f^{*} \lambda_{n}$ vanishes or trivialized so that the bundle $f$ is lifted to $\hat{f}$. This lifting of $f$ to $\hat{f}$ is also called the trivialization of the class $\lambda_{n}$.

\footnotetext{
${ }^{1}$ Previously we denoted this by $G\langle n\rangle$ but for ease of notation we drop the extra decoration here.
} 
Indeed, the pullback diagram eq. (2.8) induces another pullback diagram classifying $\widehat{G}$-principal bundles over $X$. Denoting the set of isomorphism classes of $G$-principal bundles over $X$ by $\operatorname{Bun}_{G}(X)$, we have a pullback diagram

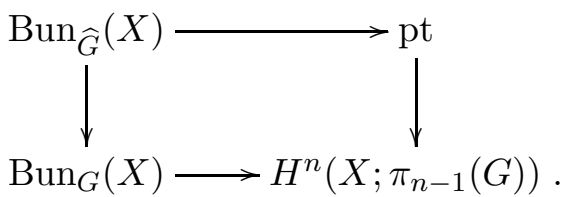

Inductively, we get a tower of connected covers which is the right side of the diagram:

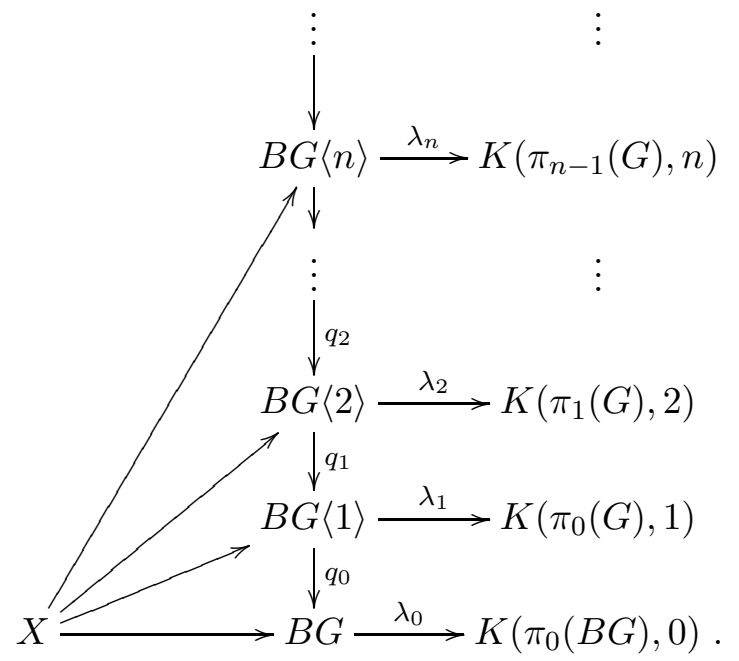

More particularly, we will be mainly interested in the case when $G\langle n\rangle$ is an $(n-1)$-connected topological group, and also in the classifying space $B G$ for some topological group $G$, i.e., $X=B G$, and its $(n-1)$-connected cover $B G\langle n\rangle$. We would like to find an $(n-2)$-connected topological group $G\langle n-1\rangle$ forming part of an $(n-2)$-connected cover $G\langle n-1\rangle \rightarrow G$ such that $B(G\langle n-1\rangle)$ is homotopy equivalent to $(B G)\langle n\rangle$.

For relatively low $2 n$ the connected covers $\mathrm{O}(k)\langle n\rangle$ are defined as the based loop spaces of the corresponding classifying spaces in [31 9] 29. It follows from the results of Kan and Milnor that every based loop space has the homotopy type of a topological group. In the homotopy category of connected $\mathrm{CW}$ complexes, there is an equivalence between loop spaces, topological groups, and associative H-spaces (see [14, Ch. 4]). Rationally, there is essentially a unique multiplication on the connected covers of the orthogonal group [32, Prop. 3].

We have the homotopy pullback diagram

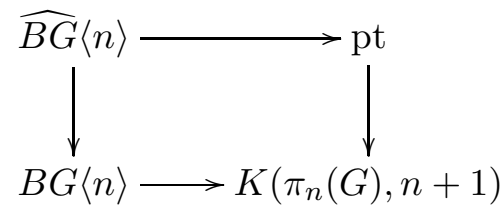

\footnotetext{
${ }^{2}$ The arguments work rationally for any $k$ [32. However in the integration from algebras to groups in the $L_{\infty}$ setting for the orthogonal case, one encounters difficulty for the $\mathbb{Z} / 2$ groups in the homotopy groups of $O$. So our discussions work fully for String and Fivebrane, which is more than what we need for this paper. For the complex/unitary case, the homotopy groups are torsion-free and so no such difficulties arise.
} 
and the fibration

$$
K\left(\pi_{n}(G), n\right) \longrightarrow \widehat{B G}\langle n\rangle \longrightarrow B G\langle n\rangle .
$$

Having a homotopy pullback diagram such as (2.15) is equivalent to saying that $\widehat{B G}\langle n\rangle$ is the homotopy fiber of $B G\langle n\rangle \rightarrow K\left(\pi_{n}(G), n+1\right)$, hence equivalent to saying that $\widehat{B G}\langle n\rangle \rightarrow B G\langle n\rangle \rightarrow$ $K\left(\pi_{n}(G), n+1\right)$ is a homotopy fiber sequence.

Next, setting $\widehat{G}\langle n-1\rangle=\Omega(\widehat{B G}\langle n\rangle)$ we have $B(\widehat{G}\langle n-1\rangle) \simeq \widehat{B G}\langle n\rangle$, and this $\widehat{G}\langle n-1\rangle$ fits into the diagram

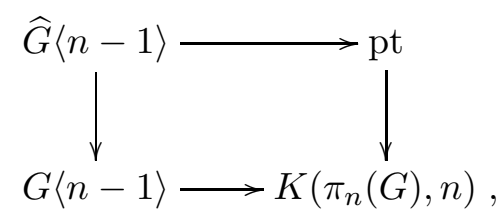

which induces a fibration with fiber $K\left(\pi_{n}(G), n-1\right)$

$$
K\left(\pi_{n}(G), n-1\right) \longrightarrow \widehat{G}\langle n-1\rangle \longrightarrow G\langle n-1\rangle .
$$

Since $K\left(\pi_{n}(G), n-1\right)$ has a group structure, then this induces (2.16) as a fiber bundle. In fact, this will a principal fiber bundle, the point being that the homotopy fiber of a map to a connected space $X$ is actually an $\Omega X$-principal bundle with the structure group an actual topological group representing the homotopy type of the loop space $\Omega X$.

For example, for the case $n=1$, we have $\widehat{G}=\operatorname{Spin}(m)$ defined in terms of Clifford algebra to be the double cover of $G=\mathrm{SO}(m), m \geq 3$.

Let $G$ be an $n$-connected topological group with $n \geq 2$. If $\pi_{n+1}(G)=A$, then there is a model for the Eilenberg-MacLane space $K(A, n)$ with the structure of a topological abelian group which forms part of an extension of topological abelian groups

$$
1 \longrightarrow K(A, n) \longrightarrow \widehat{G} \stackrel{\rho}{\longrightarrow} G \longrightarrow 1 .
$$

Moreover, $\widehat{\rho}: \widehat{G} \rightarrow G$ is a principal $K(A, n)$-bundle. This can be proved similarly to the $n=2$ case in [34], by choosing a model for $K(A, n)$ as an abelian topological group and letting $P \rightarrow G$ denote the principal $K(A, n)$-bundle classified by the fundamental class of $H^{n+1}(G, A)$. Then define $\widehat{G}$ as in [34] so that we have a short exact sequence of topological groups

$$
1 \longrightarrow \operatorname{Gauge}(P) \longrightarrow \widehat{G} \longrightarrow G \longrightarrow 1 \text {. }
$$

where Gauge $(P)$ is the gauge group of the bundle $P$. Similarly to [34] one can show that the canonical evaluation homomorphism $\operatorname{Gauge}(P) \rightarrow K(A, n)$ is a homotopy equivalence. In summary, we have:

Proposition 2.8 The fibrations in the Whitehead tower (hence also the tower of connected covers) of a Lie group are principal fiber bundles.

Note that a detailed treatment of the Whitehead tower in the rational case is given in [32], where the group structures are also identified. 


\section{Applications}

\subsection{Special orthogonal groups $\mathrm{SO}(n)$ and $\mathrm{SO}(p, q)$}

We now concentrate on the orthogonal group and its connected covers. We start with the definite signature and then work our way to indefinite signatures. Starting with the former, we have the statement that identifies when the shift in rank is inconsequential

$$
\pi_{i}(\mathrm{O}(n)) \cong \pi_{i}(\mathrm{O}(n+1)) \quad \text { for } 0<i \leq n-2 .
$$

Note that according to Kervaire [15, this cannot be improved in general as $\pi_{6}(\mathrm{SO}(7)) \cong 0$, while $\pi_{7}(\mathrm{SO}(7)) \cong \mathbb{Z}$. The following isomorphism shows that the special orthogonal group $\mathrm{SO}(n)$ has the same homotopy groups as the orthogonal group $\mathrm{O}(n)$ in positive degrees

$$
\pi_{i}(\mathrm{SO}(n))= \begin{cases}0 & \text { for } i=0 \\ \pi_{i}(\mathrm{O}(n)) & \text { for } i \geq 1\end{cases}
$$

We also note the following low degree identifications, which are often useful in calculations and applications: $\mathrm{O}(1) \cong S^{0}, \mathrm{SO}(2) \cong S^{1}, \mathrm{SO}(3) \cong \mathbb{R} P^{3}$, while $\mathrm{SO}(4)$ is the double cover of $\mathrm{SO}(3) \times$ $\mathrm{SO}(3)$, i.e., $\mathrm{SO}(4) \cong\left(S^{3} \times S^{3}\right) / \mathbb{Z}_{2}$. Note that $\operatorname{Spin}(4) \cong S^{3} \times S^{3}$.

Collecting all the above observations and results gives the following table of unstable homotopy groups of lower dimensional orthogonal groups (see e.g. [15]):

\begin{tabular}{|l||ccccccccc|}
\hline & $\mathrm{O}(1)$ & $\mathrm{O}(2)$ & $\mathrm{O}(3)$ & $\mathrm{O}(4)$ & $\mathrm{O}(5)$ & $\mathrm{O}(6)$ & $\mathrm{O}(7)$ & $\mathrm{O}(8)$ & $\mathrm{O}(9)$ \\
\hline$\pi_{0}$ & $\mathbb{Z} / 2$ & $\mathbb{Z} / 2$ & $\mathbb{Z} / 2$ & $\mathbb{Z} / 2$ & $\mathbb{Z} / 2$ & $\mathbb{Z} / 2$ & $\mathbb{Z} / 2$ & $\mathbb{Z} / 2$ & $\mathbb{Z} / 2$ \\
$\pi_{1}$ & 0 & $\mathbb{Z}$ & $\mathbb{Z} / 2$ & & & & & & \\
$\pi_{2}$ & 0 & 0 & 0 & 0 & & & & & \\
$\pi_{3}$ & 0 & 0 & $\mathbb{Z}$ & $\mathbb{Z} \times \mathbb{Z}$ & $\mathbb{Z}$ & & & & \\
$\pi_{4}$ & 0 & 0 & $\mathbb{Z} / 2$ & $\mathbb{Z} / 2 \times \mathbb{Z} / 2$ & $\mathbb{Z} / 2$ & 0 & & & \\
$\pi_{5}$ & 0 & 0 & $\mathbb{Z} / 2$ & $\mathbb{Z} / 2 \times \mathbb{Z} / 2$ & $\mathbb{Z} / 2$ & $\mathbb{Z}$ & 0 & & \\
$\pi_{6}$ & 0 & 0 & $\mathbb{Z} / 12$ & $\mathbb{Z} / 12 \times \mathbb{Z} / 12$ & 0 & 0 & 0 & 0 & \\
$\pi_{7}$ & 0 & 0 & $\mathbb{Z} / 2$ & $\mathbb{Z} / 2 \times \mathbb{Z} / 2$ & $\mathbb{Z}$ & $\mathbb{Z}$ & $\mathbb{Z}$ & $\mathbb{Z} \times \mathbb{Z}$ & $\mathbb{Z}$ \\
\hline
\end{tabular}

where the boxed entries indicate that the corresponding homotopy groups are being stabilized.

We now consider the corresponding classifying spaces. We know that the first cohomology $H^{1}(B \mathrm{O}(n), \mathbb{Z} / 2) \cong \mathbb{Z} / 2$ is generated by the first Stiefel-Whitney class $w_{1}$, so that we may pullback $w_{1}: B \mathrm{O}(n) \rightarrow K(\mathbb{Z} / 2,1)$ to obtain a 0 -connected cover, say $G$, of $\mathrm{O}(n)$ :

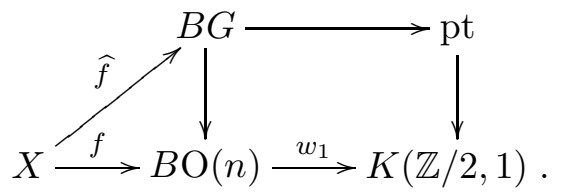

We also know that the special orthogonal group $\mathrm{SO}(n)$ has the same homotopy groups as $G$ so we may regard the connected identity component $\mathrm{SO}(n)$ of $\mathrm{O}(n)$ as the 0 -connected cover $G$. The pattern continues to the Spin group and beyond, as in [30]. 
Next, consider our main object which is the indefinite orthogonal group $\mathrm{O}(p, q)$. This has a maximal compact subgroup $\mathrm{O}(p) \times \mathrm{O}(q)$. The inclusion $\mathrm{SO}(p) \times \mathrm{SO}(q) \hookrightarrow \mathrm{SO}(p, q)$ is a homotopy equivalence by the Cartan decomposition of noncompact Lie groups, i.e. the homeomorphism from $\mathrm{SO}(p, q) \rightarrow \mathrm{SO}(p) \times \mathrm{SO}(q) \times \mathbb{R}^{p q}$.

With this observation, we can reduce the problem of connected covers in this indefinite signature setting to essentially two copies of the problem in the definite signature case. From the results in the previous section, we may pullback $w_{1} \times w_{1}: B \mathrm{O}(p) \times B \mathrm{O}(q) \rightarrow K(\mathbb{Z} / 2,1) \times K(\mathbb{Z} / 2,1)$ to obtain the 0-connected cover $G$ :

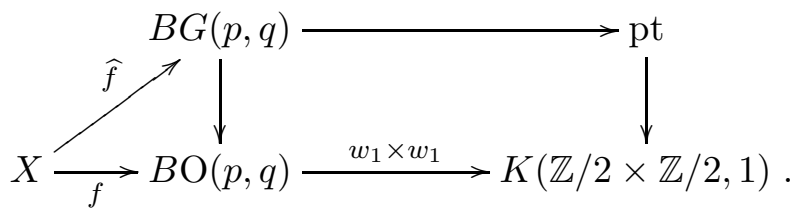

We have $G(p, q) \simeq \mathrm{SO}(p) \times \mathrm{SO}(q)$ and this is homotopy equivalent to the identity component $\mathrm{SO}(p, q)^{0}$ of $\mathrm{SO}(p, q)$, so we may also take $G=\mathrm{SO}(p, q)^{0}$ and denote this by $\widehat{\mathrm{SO}}(p, q)$ for notational consistency. Note that $\mathrm{SO}(1) \simeq$ pt and $\widehat{\mathrm{SO}}(1, n) \simeq \mathrm{SO}(n)$ so that we do not have to consider the case when $n=1$ in killing its higher homotopy groups.

On the other hand, we have the following.

Definition 3.1 The twisted covering, denoted by $\widetilde{S O}(p, q)$, is the pullback:

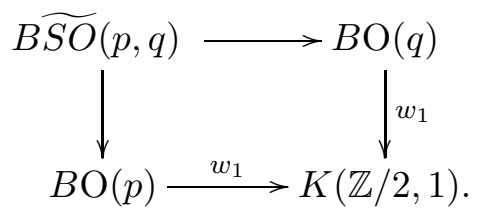

\subsection{Indefinite Spin groups}

The next step in going from $\mathrm{SO}(n)$ to $\operatorname{Spin}(n)$ by taking a double cover, or killing the fundamental group, leads to the isomorphism

$$
\pi_{i}(\operatorname{Spin}(n))= \begin{cases}0 & \text { for } i \geq 2 \\ \pi_{i}(\mathrm{O}(n)) & \text { for } i \geq 3\end{cases}
$$

Note the following useful isomorphisms in low degrees: $\operatorname{Spin}(2) \cong \mathrm{U}(1), \operatorname{Spin}(3) \cong \mathrm{SU}(2), \operatorname{Spin}(5) \cong$ $S p(2)$, and $\operatorname{Spin}(6) \cong \mathrm{SU}(4)$.

We next consider the indefinite orthogonal group $\mathrm{SO}(p, q)$ and kill the first homotopy group $\pi_{1}$. Here there are two case, depending on whether one of the factors $p$ or $q$ is greater than 1. Thus we would like to kill the fundamental group of either $\mathrm{SO}(1, n) \simeq \mathrm{O}(n)\langle 1\rangle \simeq \mathrm{SO}(n)$ or $\mathrm{SO}(p, q) \simeq \mathrm{O}(p, q)\langle 1\rangle$ for $p, q \geq 2$. An important distinction with the definite case is that the maximal compact subgroup of $\mathrm{SO}(p, q)$ is $\mathrm{SO}(p) \times \mathrm{SO}(q)$, signalling that connectedness involves more than just the usual $\mathbb{Z} / 2$. 
Remark 4 (i). The group $\operatorname{Spin}(p, q)$ is defined to be the double cover of $\mathrm{SO}(p, q)$ in a slightly nontrivial way, namely the cover corresponding to the diagonal $\mathbb{Z} / 2$ inside $\mathbb{Z} / 2 \times \mathbb{Z} / 2$. This diagonal $\mathbb{Z} / 2$ is formed of a pair of elements, where the first correspond to the kernel of $\operatorname{Spin}(p) \rightarrow \operatorname{SO}(p)$ and the second to $\operatorname{Spin}(q) \rightarrow \mathrm{SO}(q)$. For example, $\operatorname{Spin}(2,2) \cong \mathrm{SL}(2, \mathbb{R}) \times \mathrm{SL}(2, \mathbb{R})$. See [45] [2] [18] 37] [38].

(ii). The condition for having a $\operatorname{Spin}(p, q)$ structure from a $\mathrm{SO}(p, q)$ structure is the separate vanishing of two second Stiefel-Whitney classes $w_{2}^{i}, i=1,2$ (see [33] for details).

Rationally, the cohomology ring of the special orthogonal group is given as

$$
H^{*}(B \mathrm{SO}(n) ; \mathbb{Q}) \cong \begin{cases}\mathbb{Q}\left[p_{1}, p_{2}, \cdots, p_{\left[\frac{n}{2}\right]}\right], & n \text { odd }, \\ \mathbb{Q}\left[p_{1}, p_{2}, \cdots, p_{\frac{n}{2}}, e\right] /\left(p_{\frac{n}{2}}-e^{2}\right), & n \text { even },\end{cases}
$$

where $p_{i}$ are the Pontrjagin classes in degree $4 i$ and $e$ is the Euler class in degree $n$. The result on the odd case is what one expects, while the even case introduces a new generator. When considering the integral case, this generator persists and, in addition, we will have other generators arising from integral lifts of the Steifel-Whitney classes, i.e. arising from applying the Bockstein on monomials in even Stiefel-Whitney classes. Since we are interested in degree four generators, the latter will not be of relevance to us.

We know that $H^{2}(B S O(n) ; \mathbb{Z} / 2) \cong \mathbb{Z} / 2$ with the second Stiefel-Whitney class $w_{2}$ as its generator. For $n=2$, the integral cohomology of $B \mathrm{SO}(2)$ is isomorphic to $\mathbb{Z}$ with a single generator 3 $\sqrt{p_{1}}$ such that $\sqrt{p_{1}} \sqrt{p_{1}}=p_{1} \in H^{4}(B \mathrm{SO}(2) ; \mathbb{Z})$ by the result of Brown on the integral cohomology ring of $B \mathrm{SO}(n)$ [5]. In general, in the integral cohomology of $B \mathrm{SO}(n)$ the square $e^{2}$ of the Euler class is the same as the Pontrjagin class in degree $4 n$. So for $n=1$, we have a generator of degree 2 given by $e=\sqrt{p_{1}}$. One can also view this as a first Chern class if one identifies $\mathrm{SO}(2)$ with $\mathrm{U}(1)$ and hence $B S O(n)$ with $\mathbb{C} P^{\infty}$, whose cohomology is given as $H^{*}\left(\mathbb{C} P^{\infty} ; \mathbb{Z}\right) \cong \mathbb{Z}[x]$, with $|x|=2$.

Therefore, we obtain a 1-connected cover $G(n)$ of $\mathrm{SO}(n)$ for $n \geq 3$, and $G(2)$ of $\mathrm{SO}(2)$, respectively, by taking pullbacks:
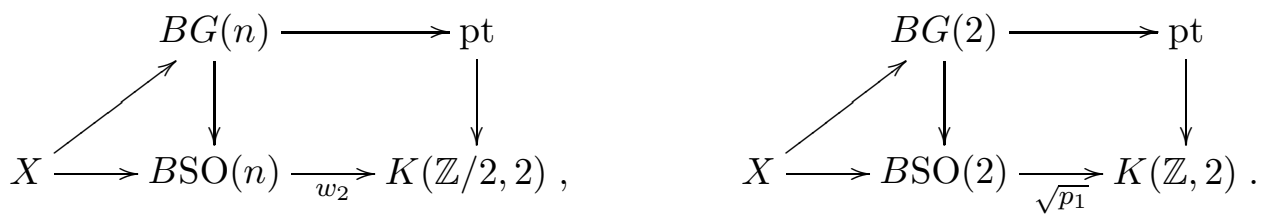

We know that the spin group $\operatorname{Spin}(n)$ is homotopy equivalent to $G(n)$ for $n \geq 3$. However, $\operatorname{Spin}(2)$ and $G(2)$ do not agree since $\operatorname{Spin}(2) \cong S^{1}$ is not simply connected.

Definition 3.2 The homotopic (definite) spin group, denoted by $\widehat{\operatorname{Spin}}(n)$, is the 1-connected cover of $\mathrm{SO}(n)$ for all $n \geq 2$ so that $\widehat{\operatorname{Spin}}(n) \simeq \operatorname{Spin}(n)$ and $\widehat{\operatorname{Spin}}(2) \simeq G(2)$.

In fact, as far as killing higher homotopy groups is concerned, $G(2)$ is trivial and we do not have to consider the case with $n=2$ in the process.

Next, in order to kill $\pi_{1}$ of $\mathrm{SO}(p, q)$, we consider three cases: When $p=q=2$, when either $p$ or $q$ is equal to 2 , and when both $p$ and $q$ are greater than 2 .

\footnotetext{
${ }^{3}$ Here we are calling the generators $\sqrt{p_{1}}$ as these square to the first Pontrjagin class. At the level of $\mathbb{Z} / 2$ coefficients, this is reminiscent of relations such as $w_{2}^{2} \equiv p_{1} \bmod 2$. In fact, this generator is the Euler class.
} 
Definition 3.3 The Spin groups in the above three cases will be the pullbacks in the following diagrams for $p, q \geq 3$ :

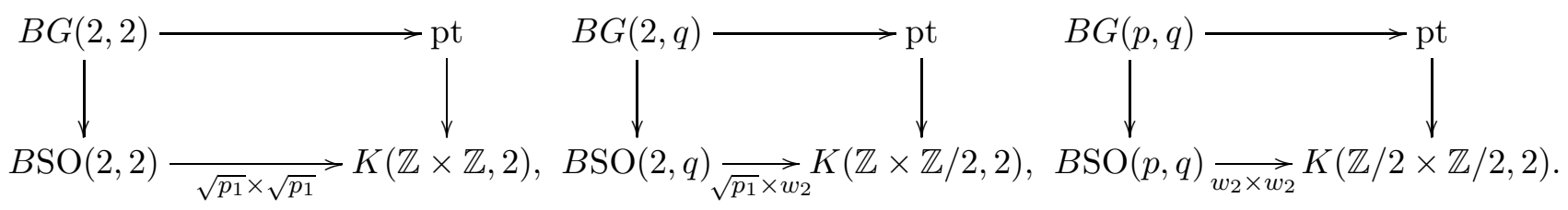

To justify this, we need to consider the cohomology groups. For that, we first need some calculations.

Lemma 3.4 The following table gives the homology groups with integral coefficient $H_{k}(B \mathrm{SO}(n) ; \mathbb{Z})$ for $k=0,1$ and 2 :

\begin{tabular}{c|ccc} 
& $n=1$ & $n=2$ & $n \geq 3$ \\
\hline$H_{0}$ & $\mathbb{Z}$ & $\mathbb{Z}$ & $\mathbb{Z}$ \\
$H_{1}$ & 0 & 0 & 0 \\
$H_{2}$ & 0 & $\mathbb{Z}$ & $\mathbb{Z} / 2$
\end{tabular}

Proof. $\mathrm{SO}(1) \cong\{1\}$ and so this case has trivial homology in nonzero degrees. Since $\mathrm{SO}(2) \cong S^{1}$, we have $B \mathrm{SO}(2) \cong \mathbb{C} P^{\infty}$. We know that

$$
H_{k}\left(\mathbb{C} P^{n} ; \mathbb{Z}\right)= \begin{cases}\mathbb{Z}, & \text { if } 0 \leq k \leq 2 n \text { and } k \text { is even, } \\ 0, & \text { otherwise. }\end{cases}
$$

so by using the direct limit $H_{k}\left(\mathbb{C} P^{\infty} ; \mathbb{Z}\right)=\lim _{n} H_{k}\left(\mathbb{C} P^{n} ; \mathbb{Z}\right)$, we obtain

$$
H_{k}\left(\mathbb{C} P^{\infty} ; \mathbb{Z}\right)= \begin{cases}\mathbb{Z}, & \text { for } k \text { even } \\ 0, & \text { for } k \text { odd }\end{cases}
$$

For $n \geq 3$, we know

$$
\pi_{k}(B \mathrm{SO}(n)) \cong \pi_{k-1}(\mathrm{SO}(n))= \begin{cases}0, & \text { if } k=1 \\ \mathbb{Z} / 2, & \text { if } k=2 .\end{cases}
$$

Hence, $H_{k}(B \mathrm{SO}(n) ; \mathbb{Z})$ follows from the Hurewicz theorem.

In order not to worry about homotopy groups in degree zero, we work with $\widehat{\mathrm{SO}}(p, q)$, the connected cover of $\mathrm{SO}(p, q)$.

Proposition 3.5 For $p, q \geq 2$, there is an isomorphism

$$
H^{2}(B \widehat{\mathrm{SO}}(p, q) ; \mathbb{Z}) \cong H^{2}(B \mathrm{SO}(p) ; \mathbb{Z}) \times H^{2}(B \mathrm{SO}(q) ; \mathbb{Z})
$$

Proof. For any positive integer $p$ and $q$, the Künneth formula gives

$$
\begin{aligned}
& H^{2}(B \mathrm{SO}(p) \times B \mathrm{SO}(q) ; \mathbb{Z}) \cong \operatorname{hom}\left(H_{2}(B \mathrm{SO}(p) \times B \mathrm{SO}(q) ; \mathbb{Z}), \mathbb{Z}\right) \\
& \oplus \operatorname{Ext}_{\mathbb{Z}}^{1}\left(H_{1}(B S O(p) \times B S O(q) ; \mathbb{Z}), \mathbb{Z}\right) .
\end{aligned}
$$


Now the homology groups inside the hom and Ext factors on the right hand side are calculated as

$$
\begin{aligned}
& H_{2}(B \mathrm{SO}(p) \times B \mathrm{SO}(q) ; \mathbb{Z}) \cong\left(\bigoplus_{r+s=2} H_{r}(B \mathrm{SO}(p) ; \mathbb{Z}) \otimes_{\mathbb{Z}} H_{s}(B \mathrm{SO}(q) ; \mathbb{Z})\right) \\
& \oplus\left(\bigoplus_{r+s=1} \operatorname{Tor}_{1}^{\mathbb{Z}}\left(H_{r}(B \mathrm{SO}(p) ; \mathbb{Z}), H_{s}(B \mathrm{SO}(q) ; \mathbb{Z})\right)\right) \\
& \cong \begin{cases}0, & \text { if } p=1, q>2 \text { or } p>2, q=1, \\
\mathbb{Z}, & \text { if } p=1, q=2 \text { or } p=2, q=1, \\
\mathbb{Z} \oplus \mathbb{Z} \cong H_{2}(B S O(p) ; \mathbb{Z}) \oplus H_{2}(B \mathrm{SO}(p) ; \mathbb{Z}), & \text { if } p, q=2 \\
\mathbb{Z} / 2 \oplus \mathbb{Z} / 2 \cong H_{2}(B S O(p) ; \mathbb{Z}) \oplus H_{2}(B S O(p) ; \mathbb{Z}), & \text { if } p, q \geq 3,\end{cases}
\end{aligned}
$$

and

$$
\begin{aligned}
H_{1}(B \mathrm{SO}(p) \times B \mathrm{SO}(q) ; \mathbb{Z}) \cong & \left(\bigoplus_{r+s=1} H_{r}(B \mathrm{SO}(p) ; \mathbb{Z}) \otimes_{\mathbb{Z}} H_{s}(B \mathrm{SO}(q) ; \mathbb{Z})\right) \\
& \oplus\left(\bigoplus_{r+s=0} \operatorname{Tor}_{1}^{\mathbb{Z}}\left(H_{r}(B \mathrm{SO}(p) ; \mathbb{Z}), H_{s}(B \operatorname{SO}(q) ; \mathbb{Z})\right)\right) \\
& =0,
\end{aligned}
$$

since $H_{1}(B \mathrm{SO}(n) ; \mathbb{Z})$ is trivial from Lemma 3.4 .

\subsection{Indefinite String groups}

In this section we take as our starting point the indefinite $\operatorname{Spin}$ group $\operatorname{Spin}(p, q)$. We emphasize that there is a subtlety here in that this group is not simply connected for general $p$ and $q$. In fact, the maximal compact subgroup of $\operatorname{Spin}(p, q)$ is $\operatorname{Spin}(p) \times \operatorname{Spin}(q) /\{(1,1),(-1,-1)\}$. The group $\operatorname{Spin}(p, q)$ itself is the diagonal 2 -fold cover of the 4 -fold cover of $\mathrm{SO}(p, q)$. For $p \geq q$, the fundamental group is given as

$$
\pi_{1}(\operatorname{Spin}(p, q))= \begin{cases}\{0\} & (p, q)=(1,1) \text { or }(1,0) \\ \{0\} & p>2, q=0,1 \\ \mathbb{Z} & (p, q)=(2,0) \text { or }(2,1) \\ \mathbb{Z} \times \mathbb{Z} & (p, q)=(2,2) \\ \mathbb{Z} & p>2, q=2, \\ \mathbb{Z} / 2 & p, q>2 .\end{cases}
$$

In order to define indefinite String structure properly we need to take as a starting point a simplyconnected group. Therefore, we should start from the simply-connected cover of $\operatorname{Spin}(p, q)$, which is what we do below. Note, however, that one can define variants of String structures without requiring this. The resulting structure would be analogous to the case of $p_{1}$-structures (see [27] [28] for various analogous structures in the definite case).

We next kill the next nontrivial homotopy groups, namely $\pi_{3}$, of the relevant Spin group $\operatorname{Spin}(p, q)$. Note that at this stage if either $p$ or $q$ is less than 3 then the corresponding factor 
in the decomposition $\operatorname{Spin}(p) \times \operatorname{Spin}(q)$ will not be seen in the process. So we will consider mainly two cases: $\operatorname{Spin}(n) \simeq \widehat{\operatorname{Spin}}(1, n) \simeq \widehat{\operatorname{Spin}}(2, n) \simeq \mathrm{O}(n)\langle 3\rangle$ and $\widehat{\operatorname{Spin}}(p, q) \simeq \mathrm{O}(p, q)\langle 3\rangle$ for $p, q \geq 3$.

When $p, q \geq 3$, the maximal compact subgroup of the 1-connected cover $\mathrm{O}(p, q)\langle 1\rangle$ is $\mathrm{O}(p)\langle 1\rangle \times$ $\mathrm{O}(q)\langle 1\rangle$ which is homotopy equivalent to $\operatorname{Spin}(p) \times \operatorname{Spin}(q)$. Hence, $\mathrm{O}(p, q)\langle 1\rangle$ is homotopy equivalent to $\operatorname{Spin}(p) \times \operatorname{Spin}(q)$. In fact, since $\mathrm{O}(1)\langle 1\rangle$ and $\mathrm{O}(2)\langle 1\rangle$ are just a point space and contractible space respectively, we still can say that $\mathrm{O}(p, q)\langle 1\rangle$ is homotopy equivalent to $\mathrm{O}(p)\langle 1\rangle \times \mathrm{O}(q)\langle 1\rangle$.

Next we would like to consider cohomology. Here the cohomology groups and rings of the orthogonal and Spin groups in the unstable range are quite subtle [5] [8] [17] [3]. However, we will only need the degree four group. Indeed, MacLaughlin has shown [21] that the fourth cohomology group is $H^{4}(B \operatorname{Spin}(n) ; \mathbb{Z}) \cong \mathbb{Z}$ and is generated by $\frac{1}{2} p_{1}$. Note that this can also be deduced from other means, for instance, from the calculations presented by Kono [17] and Benson and Wood [3].

We now get back to the construction of indefinite String structures. We obtain a 3-connected cover $G(n)$ for $n \geq 3$ by taking homotopy pullbacks, contrasting two cases:
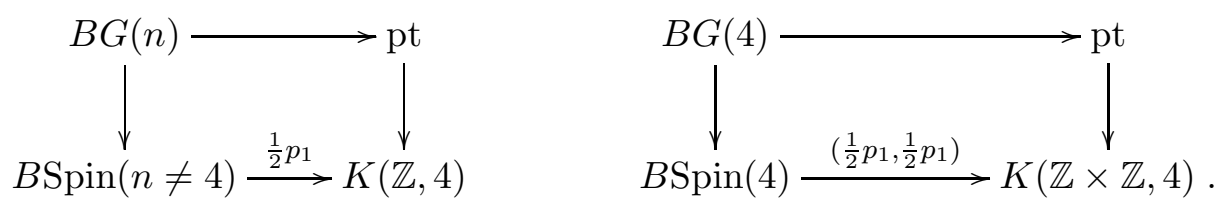

Here, the map $\left(\frac{1}{2} p_{1}, \frac{1}{2} p_{1}\right): B \operatorname{Spin}(4) \rightarrow K(\mathbb{Z} \times \mathbb{Z}, 4)$ is equivalent to the map $\frac{1}{2} p_{1} \times \frac{1}{2} p_{1}: B \operatorname{Spin}(3) \times$ $B \operatorname{Spin}(3) \rightarrow K(\mathbb{Z} \times \mathbb{Z}, 4)$ through the accidental isomorphism $\operatorname{Spin}(4) \cong \operatorname{Spin}(3) \times \operatorname{Spin}(3)$. Consequently, any classifying map $4: X \rightarrow B \widehat{\operatorname{Spin}}(3,3)$ can be decomposed into a pair $f=\left(f_{1}, f_{2}\right)$ with $f_{1}, f_{2}: B \operatorname{Spin}(3) \rightarrow K(\mathbb{Z}, 4)$. Recall also that for any classifying map $f: X \rightarrow \widehat{\operatorname{Spin}}(p, q)$ can be decomposed into $\left(f_{1}, f_{2}\right): X \rightarrow B \widehat{\operatorname{Spin}}(p) \times B \widehat{\operatorname{Spin}}(q)$, due to the homotopy equivalence of the target spaces. Hence we can use the additive and multiplicative notations interchangeably.

In order to kill $\pi_{3}$ of $\widehat{\operatorname{Spin}}(p, q)$, we take the pullbacks according to the following procedure.

Definition 3.6 The String groups in the indefinite case are defined as the loop spaces of the corresponding classifying spaces, which in turn are defined via the following pullbacks:

(i) for $p, q \geq 5$

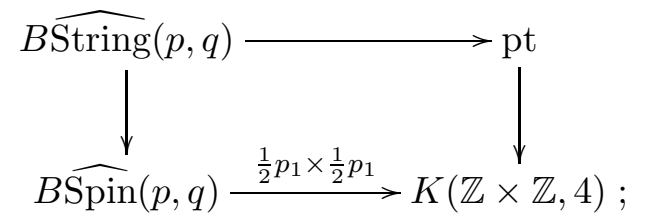

(ii) for $p=4, q \geq 5$,

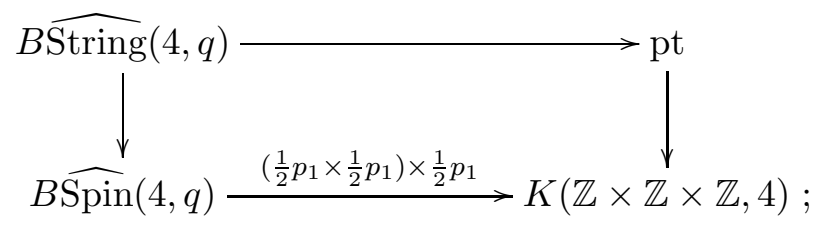

\footnotetext{
${ }^{4}$ Here a hat on the group indicates that we are taking the simply connected cover.
} 
(iii) and for $p=q=4$,

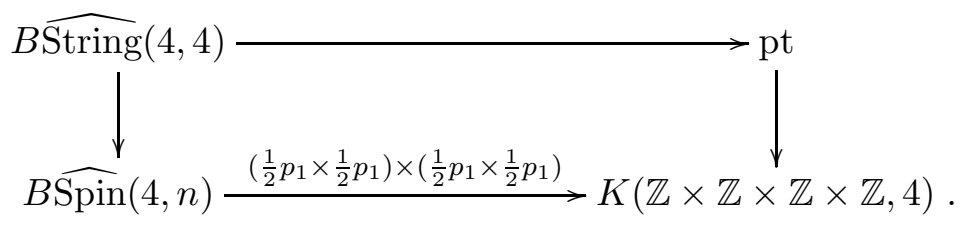

Note that we can also consider variants of String structures associated to the non-simply connected groups $\operatorname{Spin}(p, q)$.

Definition 3.7 $A\left(p_{1}, p_{1}^{\prime}\right)$-structure is a lift from $B \operatorname{Spin}(p, q)$ to the classifying space obtained by killing the fourth homotopy group.

These are analogs of a $p_{1}$-structure, where the lower homotopy groups are not necessarily killed. See [27] [28] [29] for extensions and applications.

Of course, we need to establish a decomposition of the corresponding cohomology groups. This in turn will require taking homomorphisms with homology. To that end, we start with the following:

\section{Lemma 3.8}

$$
H_{4}(B \operatorname{Spin}(p) \times B \operatorname{Spin}(q) ; \mathbb{Z}) \cong \begin{cases}H_{4}(B \operatorname{Spin}(q) ; \mathbb{Z}) & \text { for any } q \text { if } p=1 \\ H_{4}(B \operatorname{Spin}(p) ; \mathbb{Z}) & \text { for any } p \text { if } q=1 \\ H_{4}(B \operatorname{Spin}(p) ; \mathbb{Z}) \oplus H_{4}(B \operatorname{Spin}(q) ; \mathbb{Z}) & \text { if } p, q \geq 2\end{cases}
$$

Proof. The Künneth formula for homology gives the following identity:

$$
\begin{aligned}
H_{4}(B \operatorname{Spin}(p) \times B \operatorname{Spin}(q) ; \mathbb{Z}) \cong & \left(\bigoplus_{r+s=4} H_{r}(B \operatorname{Spin}(p) ; \mathbb{Z}) \otimes_{\mathbb{Z}} H_{s}(B \operatorname{Spin}(q) ; \mathbb{Z})\right) \\
& \oplus\left(\bigoplus_{r+s=3} \operatorname{Tor}_{1}^{\mathbb{Z}}\left(H_{r}(B \operatorname{Spin}(p) ; \mathbb{Z}), H_{s}(B \operatorname{Spin}(q) ; \mathbb{Z})\right)\right) .
\end{aligned}
$$

Since $H_{s}(B \operatorname{Spin}(q) ; \mathbb{Z})=0$ for $s=1,2,3$, the only nontrivial term in Tor is

$$
\operatorname{Tor}_{1}^{\mathbb{Z}}\left(H_{3}(B \operatorname{Spin}(p) ; \mathbb{Z}), H_{0}(B \operatorname{Spin}(q) ; \mathbb{Z})\right) \text {. }
$$

This is also trivial for $p \geq 2$. Moreover, when $p=1$, we have $\operatorname{Tor}_{1}^{\mathbb{Z}}(\mathbb{Z} / 2, \mathbb{Z})$ and this is trivial since $\mathbb{Z}$ is torsion-free.

The direct sum term on the right hand side of the above Künneth formula has only two nontrivial factors: $H_{0}(B \operatorname{Spin}(p) ; \mathbb{Z}) \otimes H_{4}(B \operatorname{Spin}(q) ; \mathbb{Z})$ and $H_{4}(B \operatorname{Spin}(p) ; \mathbb{Z}) \otimes H_{0}(B \operatorname{Spin}(q) ; \mathbb{Z})$. When $p \geq 2$, these two are isomorphic to $\mathbb{Z} \oplus \mathbb{Z} \cong \mathbb{Z}$. At this stage, there seems to be several routes to take. We have the isomorphisms

$$
\begin{aligned}
& H_{0}(B \operatorname{Spin}(p) ; \mathbb{Z}) \otimes H_{4}(B \operatorname{Spin}(q) ; \mathbb{Z}) \cong H_{4}(B \operatorname{Spin}(q) ; \mathbb{Z}), \\
& H_{4}(B \operatorname{Spin}(p) ; \mathbb{Z}) \otimes H_{0}(B \operatorname{Spin}(q) ; \mathbb{Z}) \cong H_{4}(B \operatorname{Spin}(p) ; \mathbb{Z}) .
\end{aligned}
$$

On the other hand, when $p=1$, we have $H_{4}(B \operatorname{Spin}(1) ; \mathbb{Z})=0$. So the only nontrivial term is now $H_{0}(B \operatorname{Spin}(p) ; \mathbb{Z}) \otimes H_{4}(B \operatorname{Spin}(q) ; \mathbb{Z}) \cong H_{4}(B \operatorname{Spin}(q) ; \mathbb{Z})$.

We will also need to calculate the Ext-term. 


\section{Lemma 3.9}

$$
\operatorname{Ext}_{\mathbb{Z}}^{1}\left(H_{3}(B \operatorname{Spin}(p) \times B \operatorname{Spin}(q) ; \mathbb{Z}), \mathbb{Z}\right) \cong \begin{cases}\mathbb{Z} / 2 & \text { if } p=1 \text { or } q=1, \\ 0 & \text { if } p, q \geq 2 .\end{cases}
$$

Proof. First, we need to compute $H_{3}(B \operatorname{Spin}(p) \times B \operatorname{Spin}(q) ; \mathbb{Z})$ and we use the Künneth formula:

$$
\begin{aligned}
H_{3}(B \operatorname{Spin}(p) \times B \operatorname{Spin}(q) ; \mathbb{Z}) \cong & \left(\bigoplus_{r+s=3} H_{r}(B \operatorname{Spin}(p) ; \mathbb{Z}) \otimes_{\mathbb{Z}} H_{s}(B \operatorname{Spin}(q) ; \mathbb{Z})\right) \\
& \oplus\left(\bigoplus_{r+s=2} \operatorname{Tor}_{1}^{\mathbb{Z}}\left(H_{r}(B \operatorname{Spin}(p) ; \mathbb{Z}), H_{s}(B \operatorname{Spin}(q) ; \mathbb{Z})\right)\right) .
\end{aligned}
$$

The Tor term is trivial since $H_{s}(B \operatorname{Spin}(q) ; \mathbb{Z})=0$ or $\mathbb{Z}$, and $\mathbb{Z}$ is torsion-free. The only nontrivial factor in the first term on the right hand side is $H_{3}(B \operatorname{Spin}(p) ; \mathbb{Z}) \otimes H_{0}(B \operatorname{Spin}(q) ; \mathbb{Z})$. This is zero for $p \geq 2$ since $H_{3}(B \operatorname{Spin}(p) ; \mathbb{Z})=0$. On the other hand, if $p=1$, we have $H_{3}(B \operatorname{Spin}(1) ; \mathbb{Z})=\mathbb{Z} / 2$ and the result follows from the relation $\mathbb{Z} / 2 \otimes \mathbb{Z} \cong \mathbb{Z} / 2$.

We are now ready to calculate the degree four cohomology groups.

Proposition 3.10 For any $p, q \geq 2$, we have

$$
H^{4}(B \operatorname{Spin}(p) \times B \operatorname{Spin}(q) ; \mathbb{Z}) \cong H^{4}(B \operatorname{Spin}(p) ; \mathbb{Z}) \oplus H^{4}(B \operatorname{Spin}(q) ; \mathbb{Z}) .
$$

For the lower dimensional case, we have

$$
H^{4}(B \operatorname{Spin}(1) \times B \operatorname{Spin}(q) ; \mathbb{Z}) \cong H^{4}(B \operatorname{Spin}(q) ; \mathbb{Z}) .
$$

Proof. The Künneth formula for cohomology asserts that

$$
\begin{aligned}
H^{4}(B \operatorname{Spin}(p) \times B \operatorname{Spin}(q) ; \mathbb{Z}) \cong & \operatorname{hom}\left(H_{4}(B \operatorname{Spin}(p) \times B \operatorname{Spin}(q) ; \mathbb{Z}), \mathbb{Z}\right) \\
& \oplus \operatorname{Ext}_{\mathbb{Z}}^{1}\left(H_{3}(B \operatorname{Spin}(p) \times B \operatorname{Spin}(q) ; \mathbb{Z}), \mathbb{Z}\right) .
\end{aligned}
$$

When $p=1$, by the above lemmas, we have

$$
\begin{aligned}
H^{4}(B \operatorname{Spin}(1) \times B \operatorname{Spin}(q) ; \mathbb{Z}) & \cong \operatorname{hom}\left(H_{4}(B \operatorname{Spin}(q) ; \mathbb{Z}) \oplus \mathbb{Z} / 2\right. \\
& \cong H^{4}(B \operatorname{Spin}(1) ; \mathbb{Z}) \times H^{4}(B \operatorname{Spin}(q) ; \mathbb{Z})
\end{aligned}
$$

Here, we used the fact that finite products and finite coproducts coincide in the additive category. When $p \geq 2$, we have

$$
\begin{aligned}
H^{4}(B \operatorname{Spin}(p) \times B \operatorname{Spin}(q) ; \mathbb{Z}) & \cong \operatorname{hom}\left(H_{4}(B \operatorname{Spin}(p) ; \mathbb{Z}) \oplus H_{4}(B \operatorname{Spin}(q) ; \mathbb{Z}), \mathbb{Z}\right) \\
& \cong \operatorname{hom}\left(H_{4}(B \operatorname{Spin}(p) ; \mathbb{Z}), \mathbb{Z}\right) \times \operatorname{hom}\left(H_{4}(B \operatorname{Spin}(q) ; \mathbb{Z}), \mathbb{Z}\right) \\
& \cong H^{4}(B \operatorname{Spin}(p) ; \mathbb{Z}) \times H^{4}(B \operatorname{Spin}(q) ; \mathbb{Z})
\end{aligned}
$$

For $p=2$, we have $H_{4}(B \operatorname{Spin}(2) ; \mathbb{Z}) \cong H_{4}\left(\mathbb{C P}^{\infty} ; \mathbb{Z}\right) \cong \mathbb{Z}$, so that $H^{4}(B \operatorname{Spin}(2) ; \mathbb{Z}) \cong \mathbb{Z}$.

The maximal compact subgroup of $G=\operatorname{Spin}(p, q)$ is $K=\operatorname{Spin}(p) \times \operatorname{Spin}(q) /(\mathbb{Z} / 2)$. Therefore, $G$ and $K$ are weakly homotopy equivalent to each other so that they are in fact homotopy equivalent, since the usual cohomology is represented by the Eilenberg-MacLane spaces in a sense that 
$H^{n}(X ; A) \cong[X, K(A, n)]$, where $A$ is a coefficient group (or an integer ring) and $X$ is arbitrary topological space. From the following short exact sequence

$$
1 \longrightarrow \mathbb{Z} / 2 \longrightarrow \operatorname{Spin}(p) \times \operatorname{Spin}(q) \longrightarrow(\operatorname{Spin}(p) \times \operatorname{Spin}(q)) /(\mathbb{Z} / 2) \longrightarrow 1
$$

and the fact that $H^{n}(\mathbb{Z} / 2 ; \mathbb{Z})=0$ for any $n$, we obtain the desired isomorphism $H^{4}(B \operatorname{Spin}(p, q) ; \mathbb{Z}) \cong$ $H^{4}(B \operatorname{Spin}(p) \times B \operatorname{Spin}(q) ; \mathbb{Z})$.

\subsection{String structure associated to indefinite unitary and symplectic groups}

The indefinite unitary group. Let $U(p, q)$ denote the group of matrices of linear isometries of the pseudo-Hermitian space $\mathbb{C}^{p, q}$ of signature $p, q$. The special indefinite unitary group $S U(p, q)=$ $\mathrm{U}(p, q) \cap \mathrm{SL}(p+q, \mathbb{C})$ is the subgroup of $U(p, q)$ consisting of matrices of determinant 1 .

The following table lists the homotopy groups of the unitary group [15] (see [20] 24] for explicit generators).

\begin{tabular}{|l|cccccc|}
\hline & $\mathrm{U}(1)$ & $\mathrm{U}(2)$ & $\mathrm{U}(3)$ & $\mathrm{U}(4)$ & $\mathrm{U}(5)$ & $\mathrm{U}(6)$ \\
\hline$\pi_{1}$ & $\mathbb{Z}$ & $\mathbb{Z}$ & $\mathbb{Z}$ & $\mathbb{Z}$ & $\mathbb{Z}$ & $\mathbb{Z}$ \\
$\pi_{2}$ & 0 & 0 & 0 & 0 & 0 & 0 \\
$\pi_{3}$ & 0 & $\mathbb{Z}$ & $\mathbb{Z}$ & $\mathbb{Z}$ & $\mathbb{Z}$ & $\mathbb{Z}$ \\
$\pi_{4}$ & 0 & $\mathbb{Z} / 2$ & 0 & 0 & 0 & 0 \\
$\pi_{5}$ & 0 & $\mathbb{Z} / 2$ & $\mathbb{Z}$ & $\mathbb{Z}$ & $\mathbb{Z}$ & $\mathbb{Z}$ \\
$\pi_{6}$ & 0 & $\mathbb{Z} / 12$ & $\mathbb{Z} / 6$ & 0 & 0 & 0 \\
$\pi_{7}$ & 0 & $\mathbb{Z} / 2$ & 0 & $\mathbb{Z}$ & $\mathbb{Z}$ & $\mathbb{Z}$ \\
\hline
\end{tabular}

Remark 5 (i) The indefinite unitary group admits a Cartan decomposition $\mathrm{U}(p, q) \cong \mathrm{U}(p) \times \mathrm{U}(q) \times$ $\mathbb{C}^{p q}$, so that - as in the case of the orthogonal group - the cohomology is determined by the maximal compact subgroup $K=\mathrm{U}(p) \times \mathrm{U}(q)$. This gives rise to two cohomology classes, one from each factor in $K$, except when $p$ or $q$ is equal to 1 , in which case there is only one class in real degree four, namely $c_{2}$ for the complementary nontrivial factor in $\mathrm{U}(1, q)$ or $\mathrm{U}(p, 1)$.

(ii) All the unitary groups have nontrivial fundamental group, isomorphic to $\mathbb{Z}$. The universal covering groups of the indefinite unitary group and the special indefinite unitary group are denoted $\widetilde{\mathrm{U}}(p, q)$ and $\widetilde{\mathrm{SU}}(p, q)$, respectively. Note that the latter is also a subgroup of the former.

(iii) As far as $\pi_{3}$ is concerned, the groups are already in the stable range. This makes the discussion much simpler than in the orthogonal case.

(iv) The cohomology rings of classifying spaces of the unitary group and the special unitary group with integral coefficients are generated by the Chern classes $c_{i}$ in degree $2 i$ and has a considerably simpler form than the orthogonal case, i.e.

$$
\begin{aligned}
H^{*}(B \mathrm{U}(n) ; \mathbb{Z}) & \cong \mathbb{Z}\left[c_{1}, c_{2}, c_{3}, \cdots, c_{n}\right] \\
H^{*}(B \mathrm{SU}(n) ; \mathbb{Z}) & \cong \mathbb{Z}\left[c_{2}, c_{3}, \cdots, c_{n}\right]
\end{aligned}
$$

String structures associated to the unitary group are considered in constructions associated to elliptic cohomology, such as in [1]. Similarly, we have: 
Definition 3.11 A String structure on a space $X$ with an indefinite unitary structure, i.e. with a map $f: X \rightarrow B \widehat{\mathrm{U}}(p, q)$ is defined via the following lifting diagram to a map $\tilde{f}$

(i) for $p, q \geq 2$

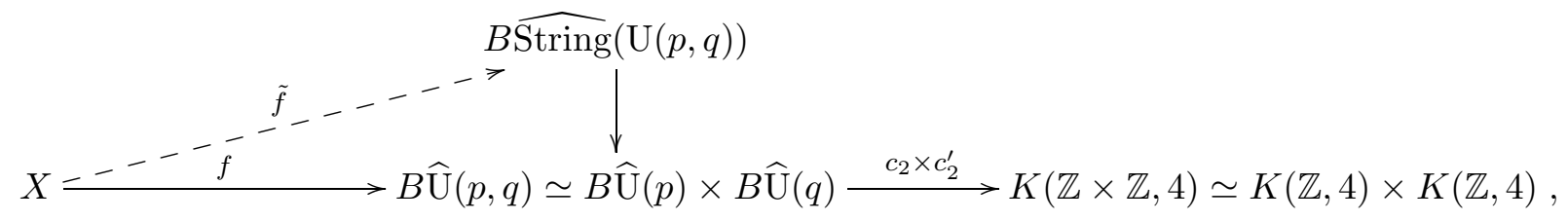

where $c_{2}, c_{2}^{\prime}:\left(E, E^{\prime}\right) \mapsto\left(c_{2}(E), c_{2}^{\prime}\left(E^{\prime}\right)\right)$ are the representatives in degree 4 of each of the factor maps;

(ii) for $p=1$

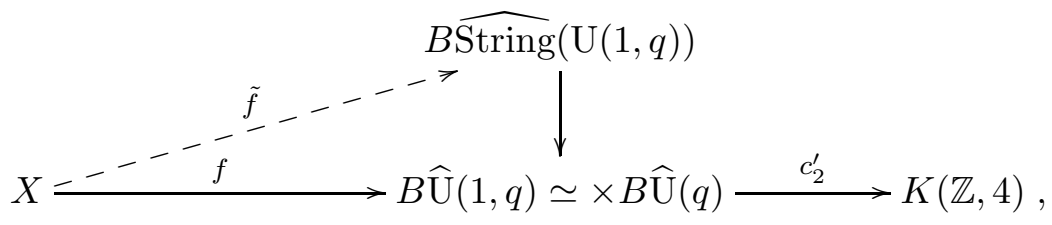

since $B \widehat{\mathrm{U}}(1) \simeq *$. Similarly for $q=1$ with a class $c_{2}$ corresponding to the first factor.

The following is immediate from the definition.

Proposition 3.12 (i) String $(\mathrm{U}(p, q))$ and $\operatorname{String}(\mathrm{SU}(p, q))$ structures are classified by a pair of classes $\left(c_{2}, c_{2}^{\prime}\right)$, where $c_{2}$ and $c_{2}^{\prime}$ are the generators in degree 4 of $B \mathrm{U}(p)$ and $B \mathrm{U}(q)$, respectively.

(ii) When either $p=1$ or $q=1$, we only have one generator as an obstruction for $\operatorname{String}(\mathrm{U}(p, q))$.

The indefinite symplectic group. The indefinite symplectic group $\operatorname{Sp}(p, q)$, also known and the indefinite quaternionic unitary group $\mathrm{U}(p, q ; \mathbb{H})$, can be defined as the isometry group of a nondegenerate quaternionic Hermitian form in $\mathbb{H}^{n}$.

Remark 6 (i) The indefinite symplectic group admits a Cartan decomposition $\operatorname{Sp}(p, q) \cong \operatorname{Sp}(p) \times$ $\operatorname{Sp}(q) \times \mathbb{H}^{p q}$, so that again the cohomology is determined by the maximal compact subgroup $K=$ $\mathrm{Sp}(p) \times \mathrm{Sp}(q)$, giving rise to two cohomology classes. Furthermore, due to the relatively large dimension, there are no degenerate cases here. For instance, $\operatorname{Sp}(1,1) \cong \operatorname{Sp}(1) \times \operatorname{Sp}(1) \cong S^{3} \times S^{3}$.

(ii) The symplectic group is simply connected, so there are no issues with the starting point to define a corresponding String structure.

(iii) $\pi_{i}(\operatorname{Sp}(n))$ is in the stable range already for $i \leq 4 n+1$. Therefore, we are in the stable range for any value of $n$ when considering the third homotopy group. The homotopy groups of the symplectic groups are computed by Mimura and Toda (see [22]).

(iv) The cohomology ring of the classifying space of the symplectic group is generated by the symplectic Pontrjagin classes $p_{i}^{\mathbb{H}}$ of degree $4 i$,

$$
H^{*}(B \operatorname{Sp}(n) ; \mathbb{Z}) \cong \mathbb{Z}\left[p_{1}^{\mathbb{H}}, p_{2}^{\mathbb{H}}, \cdots, p_{n}^{\mathbb{H}}\right]
$$

(v) Under the identification $\mathrm{Sp}(1) \cong \mathrm{SU}(2), p_{1}^{\mathbb{H}}$ is equal to $-c_{2}$. 
Definition 3.13 A String structure for the indefinite symplectic group $\operatorname{Sp}(p, q)$ on a space $X$ with classifying map $f: X \rightarrow B \widehat{\operatorname{Sp}}(p, q)$ is defined as the lifting $\tilde{f}$ in the diagram

$$
X \underset{n_{f}}{\longrightarrow} B \widehat{\operatorname{Spp}}(p, q) \simeq B \widehat{\operatorname{Spp}}(p) \times B \widehat{\operatorname{Sp}}(q) \stackrel{p_{1}^{\mathbb{H}} \times p_{1}^{\prime \mathbb{H}}}{\longrightarrow} K(\mathbb{Z} \times \mathbb{Z}, 4) \simeq K(\mathbb{Z}, 4) \times K(\mathbb{Z}, 4),
$$

where $p_{1}^{\mathbb{H}} \times p_{1}^{\prime \mathbb{H}}:\left(E, E^{\prime}\right) \mapsto\left(p_{1}^{\mathbb{H}}(E), p_{1}^{\prime \mathbb{H}}\left(E^{\prime}\right)\right)$ are the representatives in degree 4 of each of the factor maps.

As in the unitary case, it follows directly from the definition that we have the following.

Proposition 3.14 String structures associated with the indefinite symplectic group are classified by a pair of symplectic Pontrjagin classes $\left(p_{1}^{\mathbb{H}}, p_{1}^{\mathbb{H}}\right)$, where the first and second are generators of $H^{4}(B \operatorname{Sp}(p) ; \mathbb{Z})$ and $H^{4}(B \operatorname{Sp}(q) ; \mathbb{Z})$, respectively.

\subsection{Relation to twisted structures}

We have seen that indefinite structures are determined homotopically by their maximal compact subgroups that are products of two compact Lie groups. The obstruction encountered involves two characteristic classes, one from each of these factor group. It is then natural to investigate how the two 'composite structures' might interact. There is another instance where a pair of cohomological structures interact in this context, namely twisted structures ([41] [31] [27] [28] [29]), to which we now explore possible connections.

We have seen that a $G$-principal bundle $f: X \rightarrow B G$ with a topological group $G$ that is homotopy equivalent to $G^{\prime} \times G^{\prime \prime}$ can be lifted to a $\widehat{G}$-principal bundle, where $\widehat{G}$ has the same homotopy type as that of $G$ except that $\pi_{n}$ killed, when both obstruction classes $f_{1}^{*} \alpha^{\prime}$ and $f_{2}^{*} \alpha^{\prime \prime}$ in $H^{n+1}\left(X ; \pi_{n}\left(G^{\prime} \times G^{\prime \prime}\right)\right)$ vanish. That is, the outer square in the following diagram commutes up to homotopy:

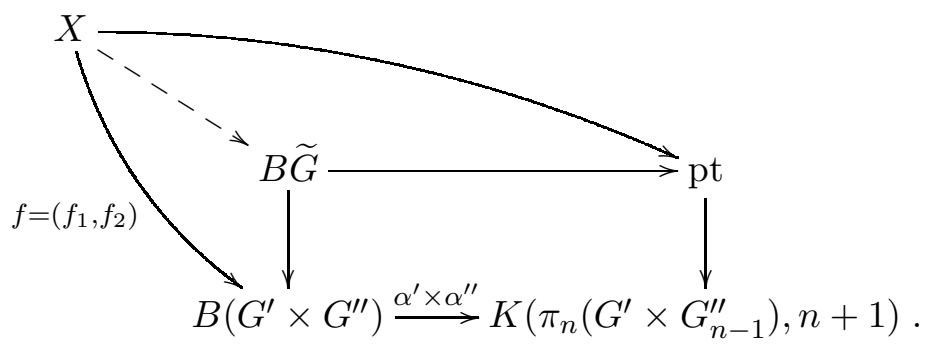

Instead of requiring both obstructions $f_{1}^{*} \alpha^{\prime}$ and $f_{2}^{*} \alpha^{\prime \prime}$ to vanish simultaneously, we may want to relax this condition to just the vanishing of the difference

$$
f_{1}^{*} \alpha^{\prime}-f_{2}^{*} \alpha^{\prime \prime}=0 \in H^{n+1}\left(X ; \pi_{n}\left(G^{\prime} \times G^{\prime \prime}\right)\right) .
$$

Now suppose the two groups $G^{\prime}$ and $G^{\prime \prime}$ have the same homotopy groups in degree $n$, i.e. we have $\pi_{n}\left(G^{\prime}\right) \cong \pi_{n}\left(G^{\prime \prime}\right)$. Denoting this isomorphism group by $\pi_{n}$, we take the pullback $\widetilde{G}_{0}$ as the following 
diagram:

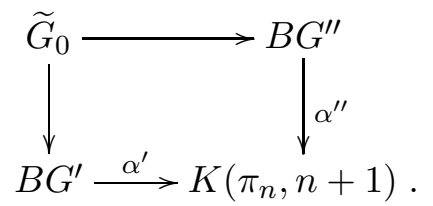

Suppose that $\widetilde{G}:=\Omega \widetilde{G}_{0}$ has a topological group structure. Given $f_{1}: X \rightarrow B G^{\prime}$ and $f_{2}: X \rightarrow B G^{\prime \prime}$ classifying $G^{\prime}$ - and $G^{\prime \prime}$-principal bundles over $X$, respectively, there is a universal $\widetilde{G}$-principal bundle over $X$ if $f_{1}^{*} \alpha^{\prime}$ is homotopic to $f_{2}^{*} \alpha^{\prime \prime}$ which is equivalent to the condition

$$
f_{1}^{*} \alpha^{\prime}-f_{2}^{*} \alpha^{\prime \prime}=0 \in H^{n+1}\left(X ; \pi_{n}\right) .
$$

Diagrammatically, the condition is equivalent to requiring that there be a homotopy $h$ as indicated in the diagram:

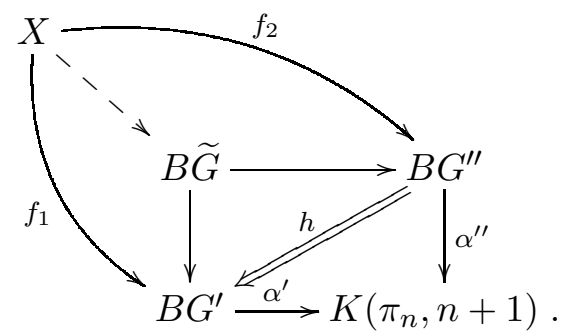

All of this motivates the following definition:

Definition 3.15 Suppose that we have two topological groups $G^{\prime}$ and $G^{\prime \prime}$ with homotopy groups $\pi_{n}^{\prime}:=\pi_{n}\left(G^{\prime}\right)$ and $\pi_{n}^{\prime \prime}:=\pi_{n}\left(G^{\prime \prime}\right)$ respectively. Moreover, suppose that we have given cohomology classes $\alpha^{\prime} \in H^{n+1}\left(B G^{\prime} ; \pi_{n}^{\prime}\right)$ and $\alpha^{\prime \prime} \in H^{n+1}\left(B G^{\prime \prime} ; \pi_{n}^{\prime \prime}\right)$ and a group homomorphism $\varphi: \pi_{n}^{\prime \prime} \rightarrow \pi_{n}^{\prime}$. Then we have the homotopy group $\widetilde{G}$ as in the previous argument, and for two $G^{\prime}$ - and $G^{\prime \prime}$-structures over $X$ given by $f_{1}$ and $f_{2}$, the induced $\widetilde{G}$-structure over $X$ is said to be twisted in favor of $G^{\prime}$ :

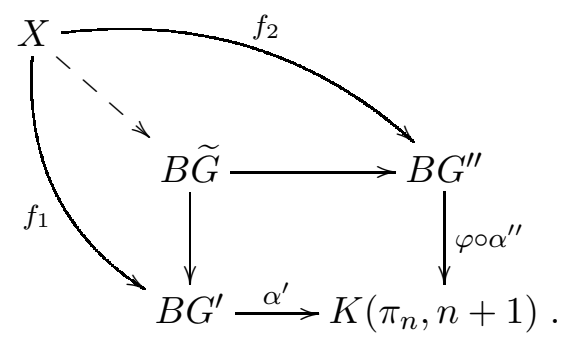

Remark 7 The twisted construction has natural motivations arising from physics, as presented by Sati-Schreiber-Stasheff [31]. For instance, the Green-Schwarz anomaly condition ([11] [10])

$$
\frac{1}{2} p_{1}(T X)-\operatorname{ch}_{2}(E)=0 \in H^{4}(X ; \mathbb{Z}),
$$

where $\operatorname{ch}_{2}(E)$ is the second Chern character of a bundle $E$ which reduces to the second Chern class $c_{2}(E)$ is equivalent to existence of a homotopy $H_{3}$ in the following diagram, with $\pi_{3}(\mathrm{SU}(n)) \cong$ 


$$
\pi_{3}(\operatorname{Spin}(n)) \cong \mathbb{Z} \text { for } n \geq 3 \text { except } 4
$$

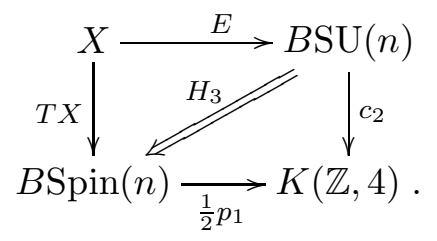

The homotopy $\mathrm{H}_{3}$ exhibits the $B$-field as a twisted gerbe, whose twist is the difference class $\frac{1}{2} p_{1}(T X)-c_{2}(E)$. Our definition above extends this to the indefinite signature case. What we have in our current context is what might essentially be viewed as a twisted String structure, in the sense of [41] [31], where the twist itself arises from a Spin bundle, where the two are the two parts in the composite maximal compact subgroup of $\operatorname{Spin}(p, q)$.

Remark 8 We have only two cases to consider for the twisted coverings:

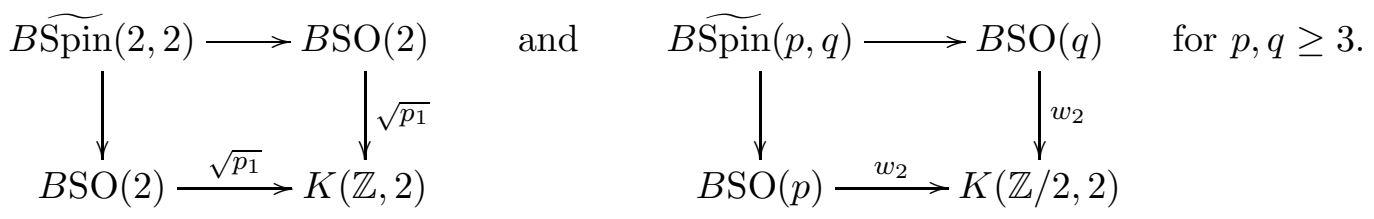

Similarly to the previous cases, we can construct twisted coverings:

Definition 3.16 The twisted covering indefinite String groups are defined as

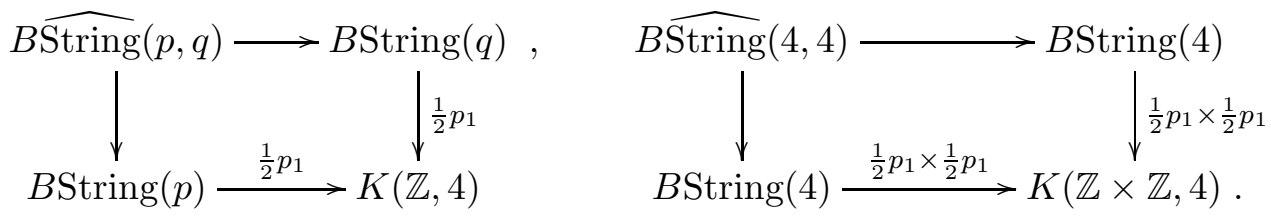

It would be very interesting to extend the definitions and constructions that we presented in this paper for String structures to include 'pseudo-Riemannian versions' of Fivebrane [30] [31] and Ninebrane structures [29]. This might require considerable calculations. We believe that it would also be worthwhile to explore geometric applications to gerbes, loop spaces, parallel transport, Chern-Simons theories, and stacky constructions, just to name a few. We hope to explore these topics elsewhere. Our initial goal was to get to these topics directly. However, we realized that seemingly straightforward matters are in fact much more subtle than meets the eye, so we believe it is worth addressing those first in this paper to provide firm ground from which to pursue further constructions.

\section{Acknowledgements}

We would like to thank Domenico Fiorenza, Corbett Redden, Jonathan Rosenberg, and Urs Schreiber for very useful discussions. The research of H. S. was supported by NSF Grant PHY1102218. We are grateful to the anonymous referee for very useful suggestions that have substantially improved the paper. 


\section{References}

[1] M. Ando, M. J. Hopkins, and N. P. Strickland, Elliptic spectra, the Witten genus and the theorem of the cube, Invent. Math. 146 (2001), no. 3, 595-687.

[2] H. Baum, Spin-Strukturen und Dirac-Operatoren über pseudoriemannschen Mannigfaltigkeiten, B.G. Teubner. Leipzig, 1981.

[3] D. J. Benson and J. A. Wood, Integral invariants and cohomology of BSpin( $n)$, Topology 34, no. 1, (1995), 13-28.

[4] A. Borel, Topics in the homology theory of fibre bundles, Lecture Notes in Mathematics 26, Springer-Verlag (1967).

[5] E. H. Brown, Jr., The cohomology of $\mathrm{BSO}_{n}$ and $\mathrm{BO}_{n}$ with integer coefficients, Proc. Amer. Math. Soc. 85, no. 2, (1982), 283-288.

[6] U. Bunke, String structures and trivialisations of a Pfaffian line bundle, Commun. Math. Phys. 307 (2011) 675, arXiv:0909.0846] [math.KT].

[7] D. Fiorenza, H. Sati, and U. Schreiber, Multiple M5-branes, String 2-connections, and 7d nonabelian Chern-Simons theory, Adv. Theor. Math. Phys. 18 (2014), 1-93, [arXiv:1201.5277].

[8] M. Feshbach, The integral cohomology rings of the classifying spaces of $\mathrm{O}(n)$ and $\mathrm{SO}(n)$, Indiana Univ. Math. J. 32 (1983), no. 4, 511-516.

[9] D. Fiorenza, U. Schreiber, and J. Stasheff, Čech cocycles for differential characteristic classes: an $\infty$-Lie theoretic construction, Adv. Theor. Math. Phys. 16 (2012), no. 1, 149250, arXiv:1011.4735] [math.AT].

[10] D. S. Freed, Dirac charge quantization and generalized differential cohomology, in Surv. Differ. Geom. VII, 129-194, Int. Press, Somerville, MA, 2000, arXiv:hep-th/0011220.

[11] M. B. Green and J. H. Schwarz, Anomaly cancellation in supersymmetric D $=10$ gauge theory and superstring theory, Phys. Lett. B 149 (1984) 117-122.

[12] A. Hatcher, Algebraic Topology, Cambridge University Press, 2002.

[13] S.-T. Hu, Homotopy Theory, Academic Press, New York, 1959.

[14] R. M. Kane, The homology of Hopf spaces, North-Holland Publishing Co., Amsterdam, 1988.

[15] M. Kervaire, Some nonstable homotopy groups of Lie groups, Illinois J. Math. 4 (1960), 161169.

[16] T. P. Killingback, Global anomalies, string theory and space-time topology, Class. Quant. Grav. 5 (1988) 1169-1186.

[17] A. Kono, On the integral cohomology of BSpin(n), J. Math. Kyoto Univ. 26-3 (1986) 333-337.

[18] H. B. Lawson, Jr. and M.-L. Michelsohn, Spin Geometry, Princeton University Press, Princeton, New Jersey, 1989. 
[19] A. Lichnerowicz, Spineurs harmoniques, C. R. Acad. Sci. Paris 257 (1963), 7-9.

[20] A. T. Lundell, Concise tables of James numbers and some homotopy of classical Lie groups and associated homogeneous spaces, Lecture Notes in Math. 1509, 250-272, Springer, Berlin 1992.

[21] D. A. McLaughlin, Orientation and string structures on loop space, Pacific J. Math. 155 (1992), 143-156.

[22] M. Mimura and H. Toda, Topology of Lie Groups, I and II, Amer. Math. Soc., Providence, RI, (1991).

[23] Th. Nikolaus, C. Sachse, and C. Wockel, A smooth model for the string group, Int. Math. Res. Not. IMRN 16 (2013), 3678-3721.

[24] T. Püttmann and A. Rigas, Presentations of the first homotopy groups of the unitary groups, Comment. Math. Helv. 78 (2003), 648-662.

[25] D. Quillen, The mod 2 cohomology rings of extra special 2-groups and the spinor groups, Math. Ann., 194 (1971), 197-212.

[26] C. Redden, String structures and canonical three-forms, Pacific J. Math. 249 (2) (2011), 447484, arXiv:0912.2086] [math.DG].

[27] H. Sati, Twisted topological structures related to M-branes II: Twisted Wu and Wu structures Int. J. Geom. Methods Mod. Phys. 09 (2012), 1250056, [arXiv:1109.4461].

[28] H. Sati, Framed M-branes, corners, and topological invariants, J. Math. Phys. 59 (2018), 062304, arXiv:1310.1060] [hep-th].

[29] H. Sati, Ninebrane structures, Int. J. Geom. Methods Mod. Phys. 12 (2015) 1550041, arXiv:1405.7686] [hep-th].

[30] H. Sati, U. Schreiber, and J. Stasheff, Fivebrane structures, Rev. Math. Phys. 21 (2009), 1197-1240, [arXiv:0805.0564] [math.AT].

[31] H. Sati, U. Schreiber, and J. Stasheff, Twisted differential string and fivebrane structures, Comm. Math. Phys. 315 (2012), no. 1, 169-213, arXiv:0910.4001] [math.AT].

[32] H. Sati and M. Wheeler, Variations of rational higher tangential structures, J. Geom. Phys. 130 (2018), 229-248, arXiv:1612.06983] [math.AT].

[33] H.-B. Shim, Indefinite string structures, Ph.D. thesis, University of Pittsburgh, 2013.

[34] S. Stolz, A conjecture concerning positive Ricci curvature and the Witten genus, Math. Ann. 304 (1996), no. 4, 785-800.

[35] S. Stolz and P. Teichner, What is an elliptic object?, in Topology, geometry and quantum field theory, 247-343, Cambridge Univ. Press, Cambridge, 2004.

[36] E. Thomas, On the cohomology groups of the classifying space for the stable spinor groups, Bol. Soc. Mat. Mexicana (2) 7 (1962) 57-69. 
[37] A. Trautman, Double covers of pseudo-orthogonal groups, Clifford Analysis and Its Applications, eds. Brace et al., 377-388, Springer, Netherlands, 2001.

[38] V. V. Varlamov, Universal coverings of the orthogonal groups, Advances in Applied Clifford Algebras 14(1) (2004), 81-168.

[39] K. Waldorf, String connections and Chern-Simons theory, Trans. Amer. Math. Soc. 365 (2013), 4393-4432, arXiv:0906.0117] [math.DG].

[40] K. Waldorf, String geometry vs. spin geometry on loop spaces, J. Geom. Phys. 97 (2015), 190-226, arXiv:1403.5656] [math.DG].

[41] B.-L. Wang, Geometric cycles, index theory and twisted K-homology, J. Noncommut. Geom. 2 (2008), no. 4, 497-552.

[42] G. Whitehead, Fiber spaces and the Eilenberg homology groups, Proc. National Acad. Sciences 38, no. 5 (1952), 426-430.

[43] G. Whitehead, Elements of homotopy theory, Springer-Verlag, New York-Berlin, 1978.

[44] E. Witten, The index of the dirac operator in loop space, in Elliptic Curves and Modular Forms in Algebraic Topology, Lecture Notes in Mathematics 1326 (1988), 161-18.

[45] J. A. Wolf, Spaces of constant curvature, Publish or Perish, Inc. Berkeley, 1977. 